\title{
ARTICLE OPEN \\ LncRNA RP11-19E11 is an E2F1 target required for proliferation and survival of basal breast cancer
}

\author{
A. Giro-Perafita (iD ${ }^{1}$, L. Luo ${ }^{1}$, A. Khodadadi-Jamayran ${ }^{2}$, M. Thompson ${ }^{1}$, B. Akgol Oksuz ${ }^{2,4}$, A. Tsirigos $\mathbb{I D}^{2,3}$, B. D. Dynlacht ${ }^{3}$, I. Sánchez ${ }^{3 *}$ and \\ F. J. Esteva ${ }^{1 *}$
}

Long non-coding RNAs (IncRNAs) play key roles in the regulation of breast cancer initiation and progression. LncRNAs are differentially expressed in breast cancer subtypes. Basal-like breast cancers are generally poorly differentiated tumors, are enriched in embryonic stem cell signatures, lack expression of estrogen receptor, progesterone receptor, and HER2 (triple-negative breast cancer), and show activation of proliferation-associated factors. We hypothesized that IncRNAs are key regulators of basal breast cancers. Using The Cancer Genome Atlas, we identified IncRNAs that are overexpressed in basal tumors compared to other breast cancer subtypes and expressed in at least $10 \%$ of patients. Remarkably, we identified IncRNAs whose expression correlated with patient prognosis. We then evaluated the function of a subset of IncRNA candidates in the oncogenic process in vitro. Here, we report the identification and characterization of the chromatin-associated IncRNA, RP11-19E11.1, which is upregulated in 40\% of basal primary breast cancers. Gene set enrichment analysis in primary tumors and in cell lines uncovered a correlation between RP11-19E11.1 expression level and the E2F oncogenic pathway. We show that this IncRNA is chromatin-associated and an E2F1 target, and its expression is necessary for cancer cell proliferation and survival. Finally, we used IncRNA expression levels as a tool for drug discovery in vitro, identifying protein kinase $C(P K C)$ as a potential therapeutic target for a subset of basal-like breast cancers. Our findings suggest that IncRNA overexpression is clinically relevant. Understanding deregulated IncRNA expression in basal-like breast cancer may lead to potential prognostic and therapeutic applications.

npj Breast Cancer (2020)6:1 ; https://doi.org/10.1038/s41523-019-0144-4

\section{INTRODUCTION}

Breast cancer is the most frequently diagnosed malignancy in women worldwide and is the second leading cause of cancerrelated death in the United States. ${ }^{1}$ Expression of the estrogen receptor (ER), progesterone receptor (PR), and amplification of the HER2 gene define the main breast cancer subtypes in terms of prognostic and therapeutic intervention. Gene expression profiling based on complementary DNA (cDNA) microarrays led to the classification of breast cancer into distinct subtypes, with separate prognostic and treatment implications: luminal A (LA), luminal B (LB), basal-like (BL), and Her2-enriched (HER2). ${ }^{2,3}$ The PAM50 assay measures the messenger RNA (mRNA) expression levels of 50 genes that can classify breast cancers into the same subtypes. Triple-negative breast cancer (TNBC), defined as lacking expression of ER/PR/HER2 receptors, represents $15-20 \%$ of breast cancer, and it is associated with the highest probability of relapse among breast cancer subtypes despite local treatments and cytotoxic chemotherapy. ${ }^{4}$ The majority of TNBCs are classified as BL and vice versa, with an overlap between the two classifications of $\sim 80 \%{ }^{5}$ The broad heterogeneity of TNBC, both inter- and intra-tumoral, has contributed to the difficulties in successfully treating it. Indeed, gene expression profiling performed in triple-negative breast cancers displayed six independent clusters with specific ontology, including two BL (BL1 and BL2), immunomodulatory (IM), mesenchymal, mesenchymal stem-like (MSL), and luminal androgen receptor $(\mathrm{LAR})^{6}$ subtypes.

With the development and improvement of genomic sequencing with high-throughput technologies, we have learned that while most of the genome is transcribed (96-98\%), 2\% of these transcripts encode for proteins. ${ }^{7}$ Although most of these noncoding transcripts have been considered junk DNA historically, in the past few decades, non-coding RNAs have been implicated in a variety of normal biological processes and disease states. ${ }^{8,9}$ Furthermore, the number of non-coding elements increases more rapidly than protein coding genes (PCG) with increasing organismal complexity. ${ }^{10}$ In addition, a high proportion of disease-related genetic variants identified with genome-wide association studies (GWAS) map to non-coding regions, suggesting a biological role for these transcripts in health and disease. ${ }^{11}$

Long non-coding RNAs (IncRNAs) are a large and diverse class of non-coding RNA transcripts with a length $\geq 200$ nucleotides. LncRNA expression has been implicated in a variety of biological processes, ranging from development and cell cycle control to apoptosis and carcinogenesis. ${ }^{8,9}$ Emerging IncRNA functional mechanisms are diverse and versatile; IncRNAs may act as guides, decoys, or scaffolds for chromatin modeling complexes, regulate post-transcriptional mRNA decay, or act as sponges for miRNA and regulate mRNA splicing, among other functions. ${ }^{12}$ We and others have shown that the IncRNA landscape in breast cancer is subtypespecific. Using unsupervised clustering analysis, IncRNA expression can classify breast cancers similarly to PCG expression. ${ }^{13,14}$ Additionally, accumulating evidence indicates that several IncRNAs are involved specifically in breast carcinogenesis. ${ }^{13,15}$

In the present study, we sought to identify clinically relevant IncRNAs deregulated specifically in basal-like breast cancer patients and then functionally evaluated a subset of these candidates in the oncogenic process in vitro and assessed their value as prognostic markers. We identified and characterized the

\footnotetext{
'Division of Hematology/Oncology, Perlmutter Cancer Center, NYU Langone Health, New York, NY, USA. ${ }^{2}$ Applied Bioinformatics Laboratories, NYU School of Medicine, New York, NY, USA. ${ }^{3}$ Department of Pathology, NYU School of Medicine, New York, NY, USA; ${ }^{4}$ Present address: Program in Systems Biology, University of Massachusetts Medical School, Worcester, USA. *email: Irma.Sanchez@nyulangone.org; fjesteva@gmail.com
} 
chromatin-associated IncRNA, RP11-19E11.1, which is upregulated in $40 \%$ of basal primary breast cancers. Gene set enrichment analysis (GSEA) in primary tumors and in cell lines uncovered a correlation between RP11-19E11.1 expression level and the E2F oncogenic pathway. We show that this IncRNA is chromatinassociated and an E2F1 target, and its expression is necessary for cancer cell proliferation. Finally, we used IncRNA expression levels as a tool for drug discovery in vitro and identified PKC as a potential therapeutic target for a subset of BL breast cancers.

\section{RESULTS}

Identification of clinically relevant IncRNAs overexpressed specifically in BL breast cancer

In order to identify IncRNAs that play a role in BL breast cancer, we used RNA-sequencing (RNA-seq) data from 1183 patients available in the The Cancer Genome Atlas (TCGA) database. We classified the tumors with available PAM50 molecular subtype annotation, ${ }^{16}$ obtaining a final cohort of 769 patients represented by 131 BL, 64 HER2, 404 LA, and 170 LB subtypes (Fig. 1a). We excluded 25 tumors initially classified as normal-like (NL) subtype for further analysis. We and others have previously shown that IncRNA expression reflects subtype specificity in breast cancer. ${ }^{13,14,17}$ Accordingly, t-SNE (t-distributed stochastic neighbor embedding) analysis of the top 500 IncRNAs expressed in patients showed a molecular subtype-based clustering for this cohort of patients, similar to the one obtained using marker genes, a mix between coding and non-coding genes (Fig. 1b, Supplementary Fig. 1a). Using differentially expressed gene analysis (DEseq analysis), we identified a subset of IncRNAs overexpressed in the BL subtype (>1.5-fold change) compared to normal tissue and other subtypes. To enrich for clinically relevant IncRNAs, we filtered out those with low baseMean $(<0.5)$ expressed in fewer than $10 \%$ of the patients. To study their function in vitro, we analyzed the RNA-seq data of a panel of cell lines and selected those IncRNAs that were expressed in at least two BL breast cancer cell lines. To further restrict the list of potential IncRNAs overexpressed in BL breast cancer, we selected the genes that were highly expressed in BL breast cancer and minimally expressed in the other subtypes (cut-off was $<15 \%$ of patients with FPKM (fragments per kilobase of exon model per million reads mapped) $>1$ ) (Fig. 1c, d). Interestingly, this produced a list of nine candidates, expression of which was sufficient to cluster the BL subtype tumors by t-SNE analysis (Supplementary Fig. 1b).

In vitro validation and functional screening of IncRNAs overexpressed in BL breast cancer

To study potential roles of these IncRNAs in tumor growth or cancer progression using in vitro models, we analyzed their levels in cell lines representing different molecular subtypes of breast cancer with Quantitative reverse transcription PCR (qRT-PCR). We selected for further studies those IncRNAs showing detectable levels by qRT-PCR and overexpression (OE) of at least ten-fold in at least two BL cell lines compared to other subtypes of cancer and/ or normal breast cell lines. LncRNAs CTD-2015G9.2, RP11-19E11.1, AC01917.1, and RP3-522D1.1 fulfilled these criteria, and since their expression patterns in cell lines mirrored those observed in patients, they were selected for further functional studies (Figs 1e and 2a). We next examined the localization of these IncRNAs as this could provide insight into their potential functions. Three of the four candidates showed mostly cytoplasmic localization (similar to glyceraldehyde 3-phosphate dehydrogenase (GAPDH)), while RP11-19E11.1 exhibited strong nuclear localization, similar to levels observed for nuclear non-coding RNA (ncRNA) controls, MALAT-1 and 7SK. (Fig. 2b). Using the patient survival data available in TGCA, we asked whether any of our candidates would behave as a prognostic marker. We divided the patients into high and low expression groups for each of the four candidates and performed log-rank test (Supplementary Fig. 2). Patients with higher levels of the IncRNA AC01917.1 had somewhat better survival profiles ( $p$ value 0.035 ), whereas patients with high levels of RP11-19E11.1 exhibited poor survival outcomes ( $p$ value 0.041 ). We then asked if any of these IncRNAs played a role in cancer cell growth and migration. To assess this, we designed small interfering RNAs (siRNAs) for the cytoplasmic IncRNAs and locked nucleic acid (LNA) GapmeRs (LNAs) to knock down the nuclear IncRNA. Initially, we measured cell viability after transcript knockdown using two different cell lines that overexpressed the IncRNA candidates (Fig. 2c, d). Of note, viability was reduced in all cell lines depleted of CTD-2015G9, RP11-19E11.1, or RP3-522D1.1, but there was no effect observed after knock-down of AC01917.1. We therefore tested whether this IncRNA might be important in cell migration and/or invasion (Fig. 2e) and found that knock-down of AC01917.2 in two different cell lines impaired migration and invasion in Boyden chamber migration assays.

Thus, our studies indicate that we have identified a small group of IncRNA candidates with BL subtype-specific expression in both patients and cell lines. Importantly, our ability to cluster BL tumors based on this profile suggests that these IncRNAs could have prognostic value and play a potential role in tumor development and carcinogenesis.

Genetic characterization of the BL-specific and chromatinassociated IncRNA RP11-19E11.1

The nuclear IncRNA, RP11-19E11.1, is overexpressed in 40\% of BL patients. We showed that cell viability was strongly impaired after knock-down and that patients with high levels of this IncRNA have a poor prognosis. Therefore, we sought to characterize its potential function in depth and focused on this IncRNA in the remainder of our study.

RP11-19E11.1 is an intragenic IncRNA. The annotation for this IncRNA identified two variants of the transcript with different $5^{\prime}$ and $3^{\prime}$ ends (Fig. 3a). In order to verify the authentic $5^{\prime}$ and $3^{\prime}$ ends of this IncRNA, we performed $5^{\prime}$ and $3^{\prime}$ rapid amplification of CDNA ends (RACE) using the nuclear fraction of a cell line that highly expresses this IncRNA (HCC2157). Our results showed that in contrast with previous annotations, variants 1 and 2 (V1 and V2) share the same $3^{\prime}$ end (Fig. 3a). Further, we were not able to detect any transcripts with the $5^{\prime}$ end of annotated V2, while two different isoforms with the same introns as V2 shared the $5^{\prime}$ end of V1 (Fig. 3a). Sequencing results of the RACE clones showed that all the variants incorporated 85 additional nucleotides at the $5^{\prime}$ end of the annotated $\mathrm{V} 1$.

We next explored whether this IncRNA is associated with chromatin, as many nuclear IncRNAs have been reported to play a role in chromatin remodeling or transcriptional regulation, among other functions. ${ }^{18,19}$ Our results clearly indicated chromatin association, similar to Neat-1 or Xist, used as controls for both V1 and V2 (Fig. 3b). Nuclear IncRNAs can regulate expression of neighboring genes in cis. To investigate this hypothesis, we plotted a heatmap for the expression of all genes found in a $1 \mathrm{Mb}$ window around the IncRNA (Fig. 3c). Strikingly, we observed a near-perfect correlation in expression between RP11-19E11.1 and EN1, a neural-specific transcription factor localized $\sim 13 \mathrm{~kb}$ away that was implicated in basal-like breast cancer. ${ }^{20}$ This correlation was also observed in multiple cell lines (Fig. 3d). However, after knock-down of the IncRNA, we did not observe a significant change in the expression of neighboring genes, including EN1 (Fig. 3e). Moreover, knock-down of EN1 did not significantly change RP11-19E11.1 expression (Supplementary Fig. 3a). We also noted that OE of both transcripts was not due to an amplification of this region on the chromosome (cBioportal). Since both promoters of RP11-19E11.1 and EN1 are enriched for CpG islands, we speculated that the observed co-OE could be due to 
A.

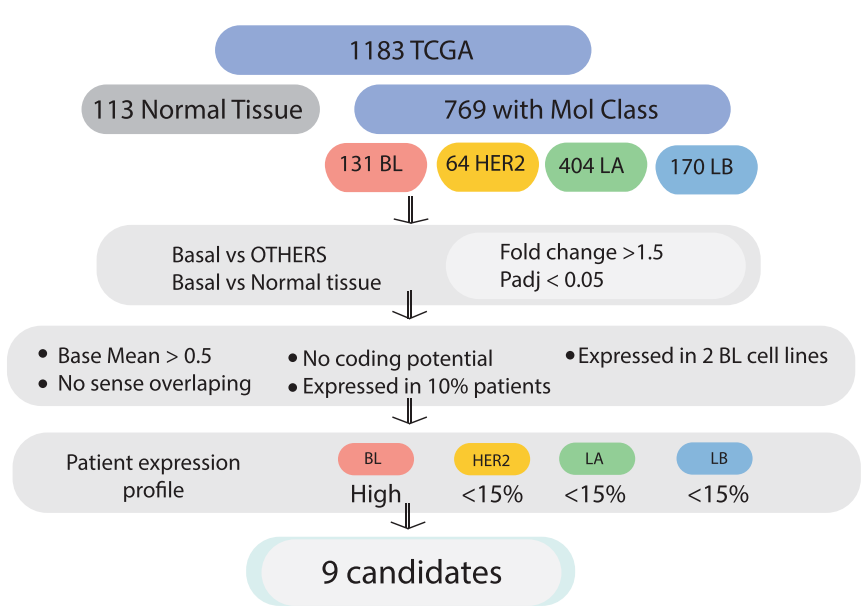

C.

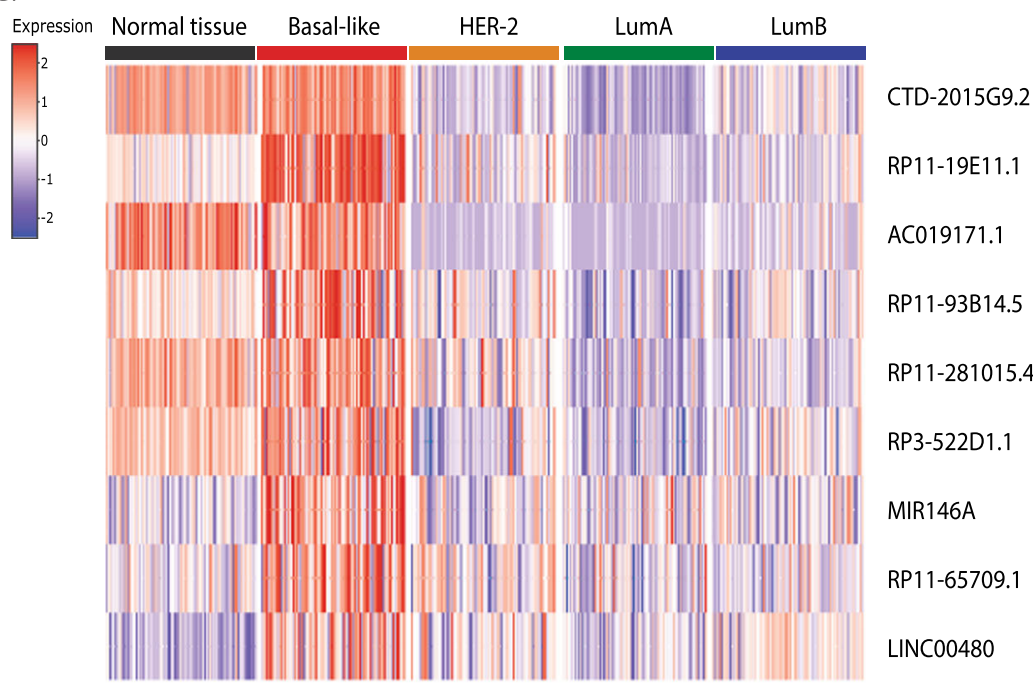

B.

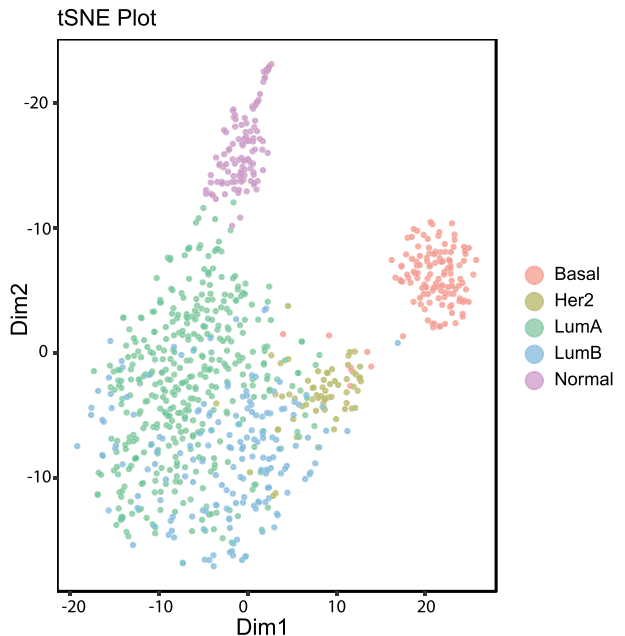

D.
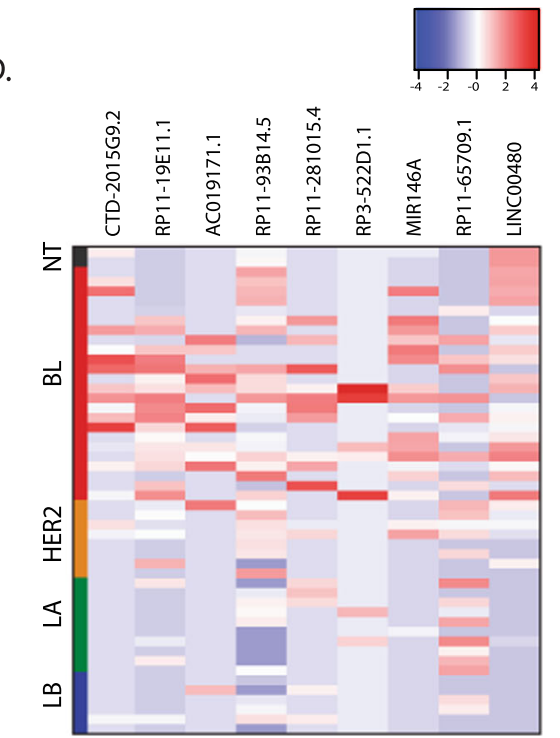

E.
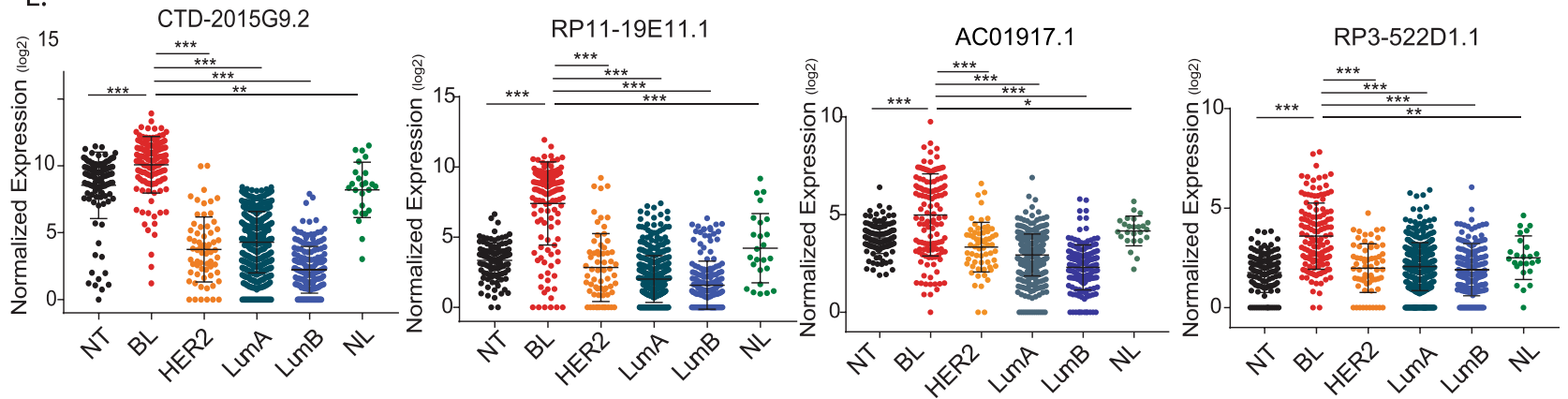

Fig. 1 Identification of IncRNAs specific for the basal-like breast cancer subtype using patient data from the TCGA database. a Pipeline flow chart used to identify InCRNA candidates. b t-SNE plot originated from the expression of the 500 top-expressed IncRNA in patients. c Heatmap showing the expression of the nine IncRNA candidates in different breast cancer subtypes. $\mathbf{d}$ Heatmap showing the expression of the nine candidates in a panel of cell lines classified according to their molecular subtype. e Dot plot showing the levels of expression for each patient within the breast cancer subtypes for the IncRNA candidates selected. Mean \pm SD are represented for each condition. ${ }^{*} P<0.05 ;{ }^{* *} p<$ $0.01 ;{ }^{* * *} p<0.001$ (ANOVA). NT normal tissue, BL basal-like, HER2 her2-enriched, LA luminal A, LB luminal B, NL normal-like.

deregulation of DNA methylation in the promoter of these two transcripts. Using TCGA methylation data, we found that the percentage of methylated CpG islands in this region was significantly lower for triple-negative patients than the other subtypes of cancer or normal tissue (Supplementary Fig. 3b). DNA methylation is an essential layer of regulation in mammalian genomes, controlling several biological processes, including development. Patterns of DNA methylation are profoundly altered in cancer, both by suppressing the transcription of tumor suppressor genes by promoter hypermethylation and by activating gene transcription due to global hypomethylation. ${ }^{21}$ Our data suggest that expression of RP11-19E11.1 could be deregulated in a subset of basal tumors as a result of hypomethylation of a large chromosomal region that includes this IncRNA. Since all 
A.
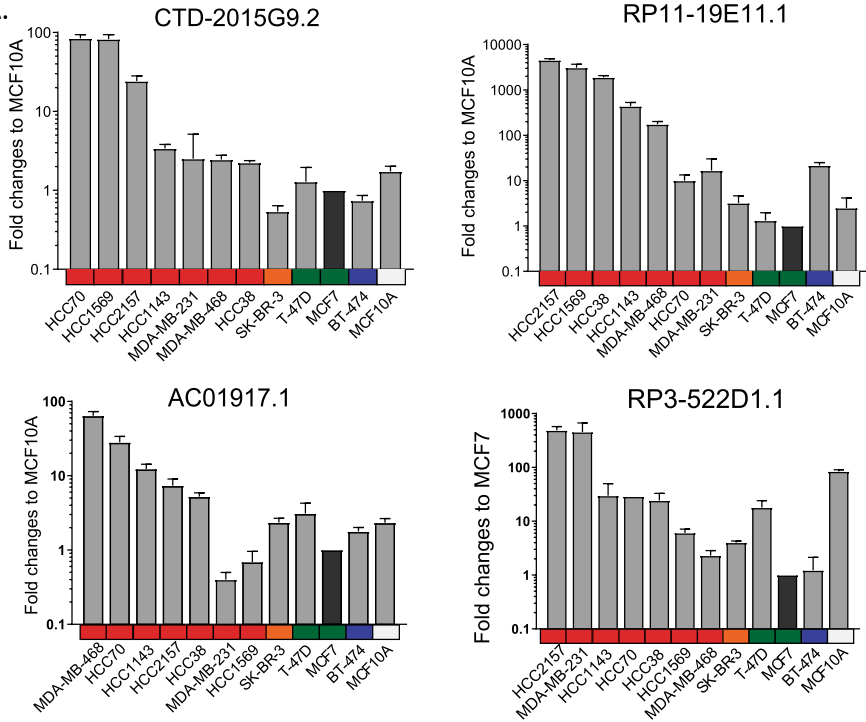

RP11-19E11.1

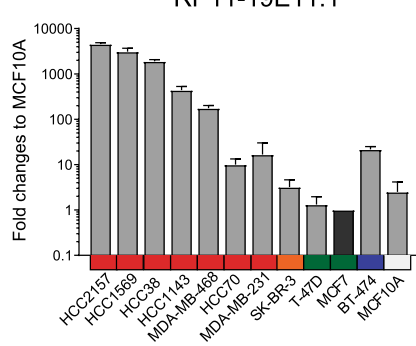

CTD-2015G9.2
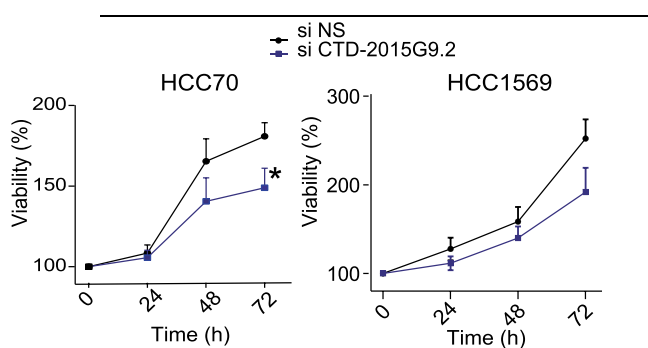

AC01719.1

-si RP11-173C1.1
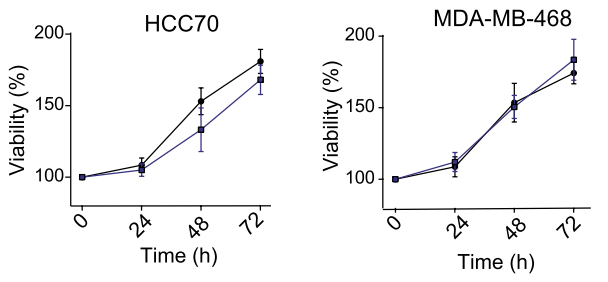

D.
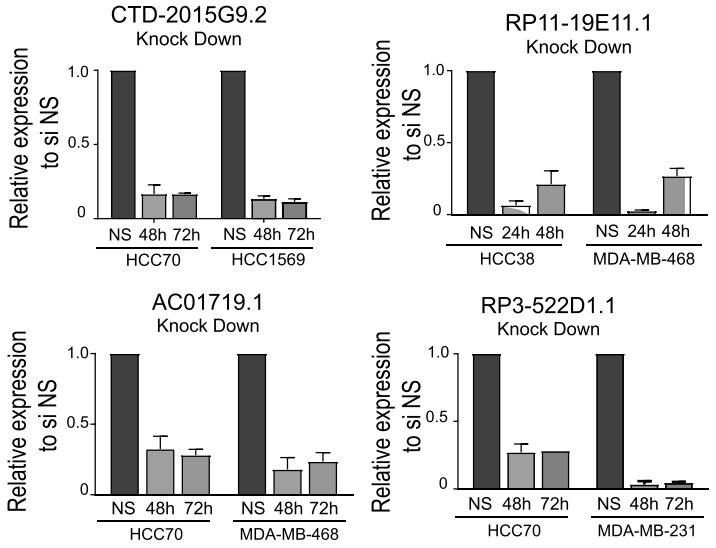

E.
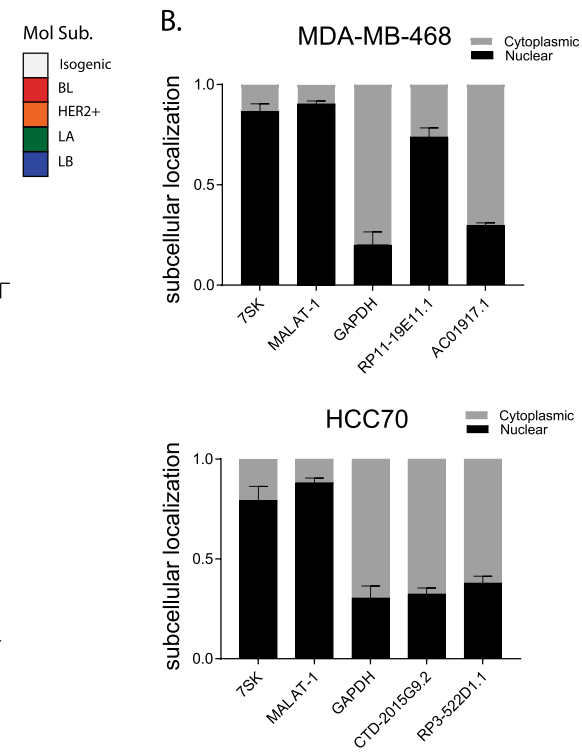

RP11-19E11.1

$\rightarrow$ - LNANS $11-19 E 1.1$
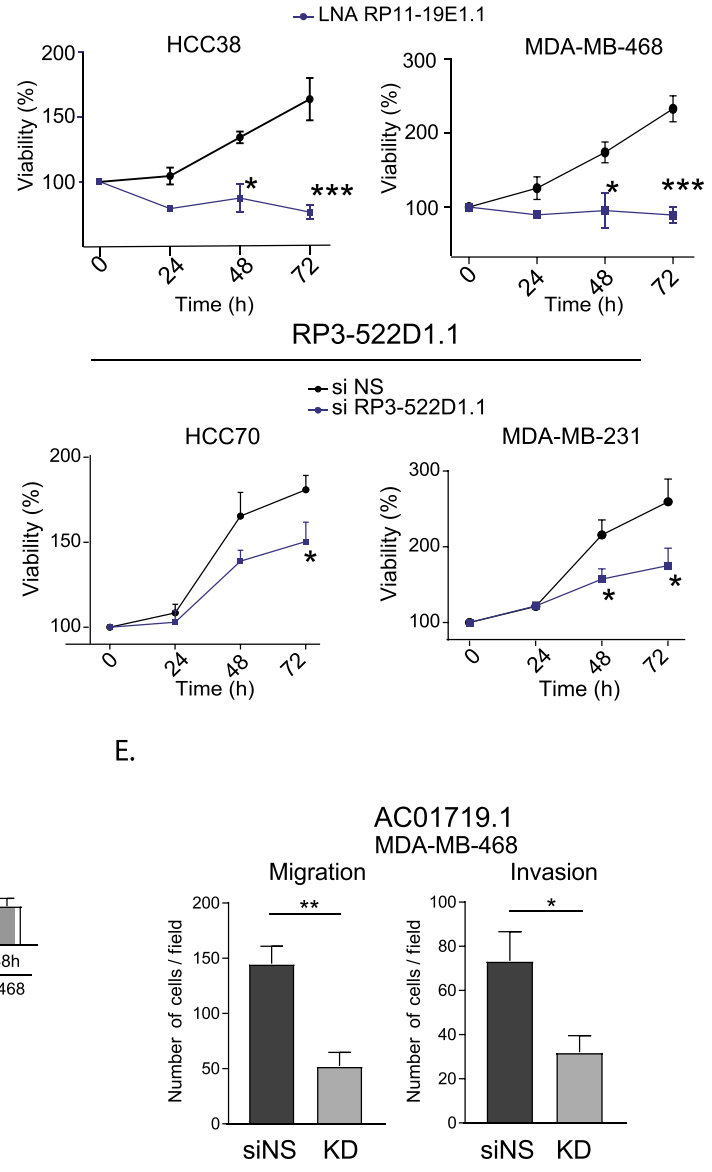

Fig. 2 In vitro validation and functional screening of IncRNA candidates. a RNA expression obtained by qRT-PCR for four of the IncRNA candidates in a panel of cell lines. MCF7 cell line was used as reference. b Subcellular localization of the IncRNA candidates assessed by qRTPCR after nuclear-cytoplasm fractionation. c Viability assays (MTT) after knock-down (siRNA 20 nM or LNA 50 nM) in two different cell lines for each of the IncRNA candidates at different time points. d RNA levels of the IncRNA candidates after knock-down in two cell lines at 48 and $72 \mathrm{~h}$ after transfection. e Migration and invasion in the MDA-MB-468 cell line after IncRNA AC01719.1 knock-down. Experiments were performed at least three times in triplicate. Data are presented as mean $\pm \mathrm{SEM}$. ${ }^{*} P<0.05 ;{ }^{* *} p<0.01 ;{ }^{* * *} p<0.001$ (two-tailed unpaired $t$ test). 


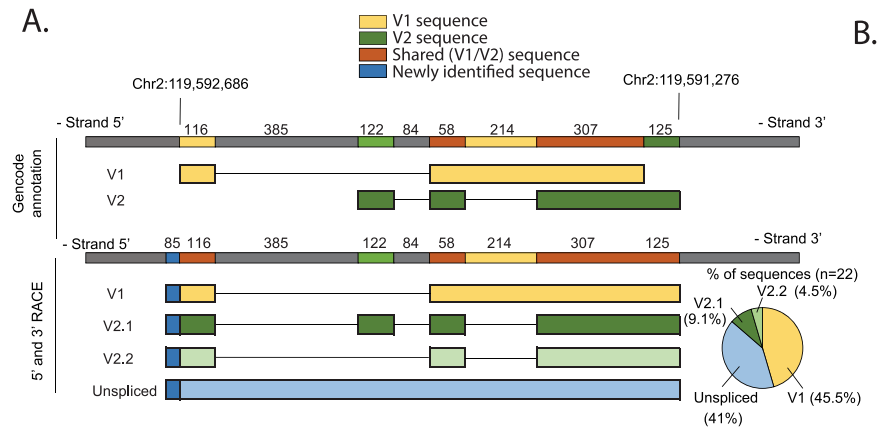

C.

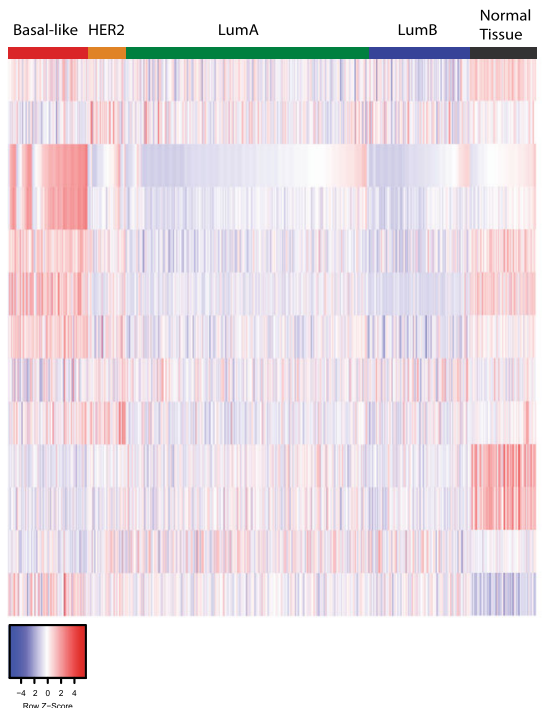

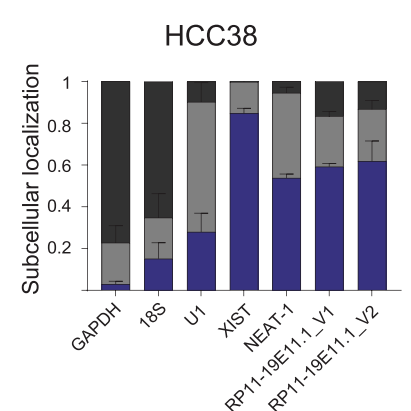

D.

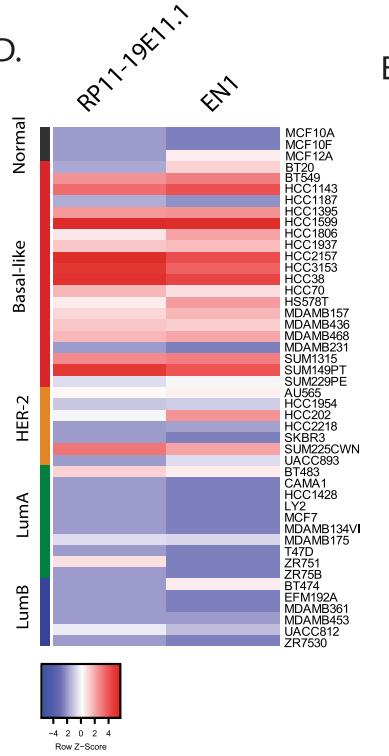

E.

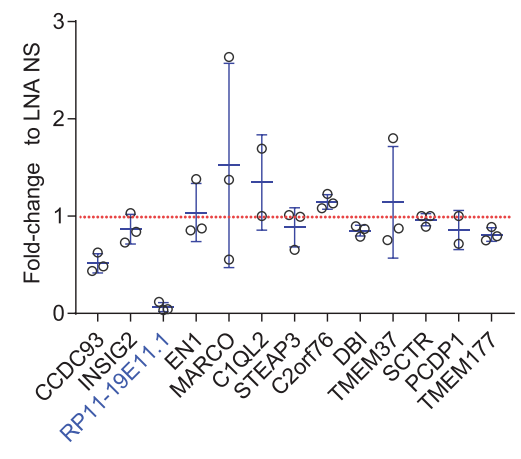

$1 \mathrm{MB}$ Neighboring genes

Fig. 3 LncRNA RP11-19E11.1 transcript characterization. a Scheme representing $5^{\prime}$ and $3^{\prime}$ RACE results for the three main variants identified. b Subcellular localization of V1 and V2 assessed by qRT-PCR after nuclear-cytoplasm fractionation and chromatin precipitation. Data are presented as mean value \pm SEM. c Heatmap of the expression of 1 Mb neighboring genes of IncRNA RP11-19E11.1 in TCGA patients. d Heatmap of the expression of RP11-19E11.1 and EN1 in a panel of cell lines. e Dot plot showing the expression levels of RP11-19E11.1 neighboring genes for each cell line. For each gene mean \pm SD is represented. Value not shown for those genes in which expression was below detection level. Experiments were performed at least three times in triplicate.

RP11-19E11.1 transcripts identified show nuclear localization and chromatin association, we propose that the function of this IncRNA is linked to regulation of chromatin-associated processes.

Patients with high RP11-19E11.1 show specific oncogenic signature

Since patients with high levels of RP11-19E11.1 have a poorer prognosis than those exhibiting lower expression, we asked if patients that overexpress RP11-19E11.1 share a specific oncogenic signature. To that end, we compared BL patients with high and low RP11-19E11.1 expression (1Q vs. $4 Q, n=33$ for each group) using GSEA (Fig. 4a). Intriguingly, patients with high levels of RP1119E11.1 showed significant downregulation of the hallmark phosphatidylinositol-3-kinase/Akt/mammalian target of rapamycin signaling pathway and upregulation of the RB/E2F oncogenic pathway (Fig. 4b, c). We then evaluated if there was any alteration in some of the principal effectors of this axis commonly deregulated in cancer (RB, E2F, CDKN2A, CCDN1, CDK4) by amplification, deletion, or mutation that could be associated with the expression pattern of the IncRNA ${ }^{22}$ (Fig. 4d). Deregulation of this pathway is known to be prevalent in breast cancer and is connected to poor outcome. ${ }^{23}$ We did not find significant differences between patients with high and low RP11-19E11.1 in the prevalence of any gene alterations that can activate these pathways (Fig. 4d, e). As patients with high RP11-19E11.1 show a specific oncogenic signature, we analyzed whether the expression of this IncRNA was restricted to a unique TNBC molecular subtype, as defined by another well-established classification scheme. ${ }^{6,24}$ The stratification of 102 TNBC patients in these six different subtypes showed a significant enrichment of RP11-19E11.1 in the BL1 subtype (Fig. $4 \mathrm{f}$ ). The BL1 is a subtype enriched in cell cycle and cell division components and pathways, showing increased proliferation and cell cycle check point loss. These results align well with our GSEA analysis. Furthermore, we also observed that this IncRNA was also highly expressed in the subtypes IM and mesenchymal, both subtypes having highly proliferative phenotypes compared to BL2, MSL, or LAR.

These results suggest that factors other than genetic background trigger the E2F oncogenic signature and that this signature correlates with poor prognosis in BL breast cancer.

IncRNA RP11-19E11.1 knock-down induces cell cycle arrest and apoptosis

We extended our analysis of a panel of BL cell lines to study the levels of both variants of the IncRNA RP11-19E11.1. We also performed viability assays (MTT, 3-(4-5-dimethylthiazol-2-yl)-2,5diphenyl tetrazolium bromide) with two different LNAs separately, targeting different regions and variants of this IncRNA in two different cell lines, HCC38 (viability at $72 \mathrm{~h}$ for LNA2 vs. LNA NS $p$ value 0.017 , for LNA3 vs. LNA NS $p$ value $<0.001$ ) and SUM149PT 
A.

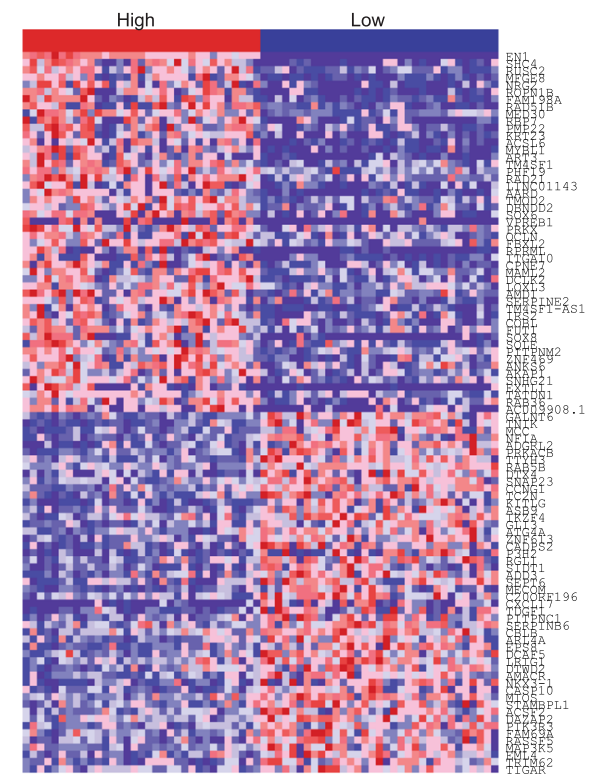

C.
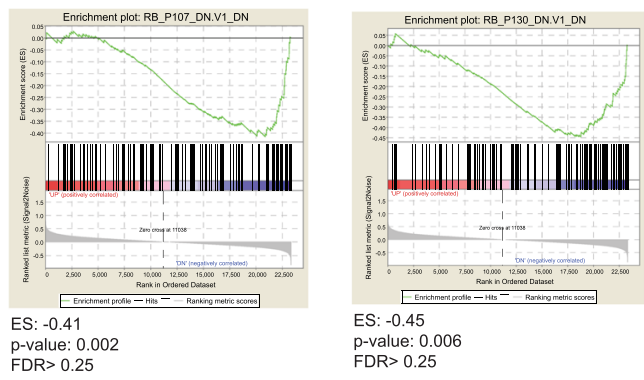

B.

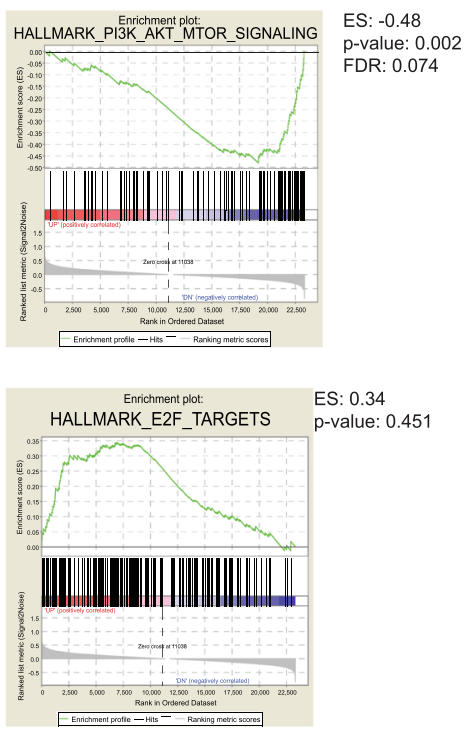

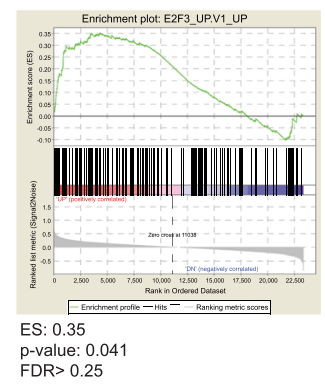

FDR $>0.25$

D.

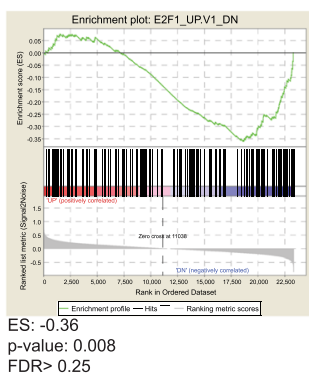

\begin{tabular}{|c|c|c|c|c|c|c|c|c|c|c|c|c|}
\hline & $\mathrm{n}$ & RB (HD) & $\mathbf{R B}(\mathrm{M})$ & E2F1 (AMP) & E2F3 (AMP) & E2F5 (AMP) & E2F6 (AMP) & E2F6 (HD) & CCND1 (AMP) & CDK4 (AMP) & CDKN2A (AMP) & CDKN2A (HD) \\
\hline $\mathrm{HIGH}$ & 33 & $12.1 \%(4)$ & $3.0 \%(1)$ & $3.0 \%(1)$ & $6.0 \%(2)$ & $18 . \%(6)$ & $3.0 \%(1)$ & $3.0 \%(1)$ & $3.0 \%(1)$ & $3.0 \%(1)$ & $0 \%(0)$ & $6.0 \%(2)$ \\
\hline LOW & 33 & $15.1 \%(5)$ & $6.0 \%(2)$ & $9.0 \%(3)$ & $6.0 \%(2)$ & $9.0 \%(3)$ & $6.0 \%(2)$ & $0 \%(0)$ & $9.0 \%(3)$ & $0 \%(0)$ & $3.0 \%(1)$ & $0 \%(0)$ \\
\hline Basal & 133 & $14.2 \%(19)$ & $4.5 \%(6)$ & $5.2 \%(7)$ & $9.0 \%(12)$ & $16.5 \%(22)$ & $6.7 \%(9)$ & $0.7 \%(1)$ & $4.5 \%(6)$ & $0.7 \%(1)$ & $0.7 \%(1)$ & $3.0 \%(4)$ \\
\hline
\end{tabular}

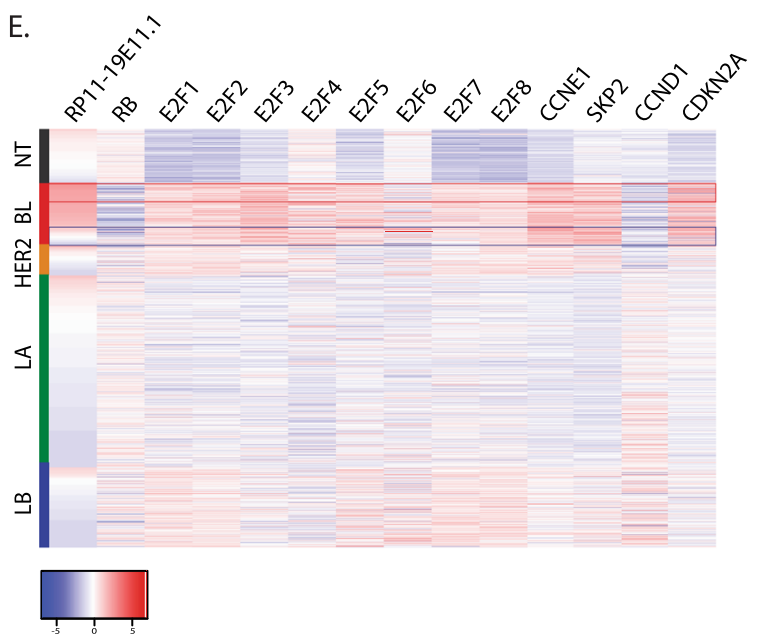

F.

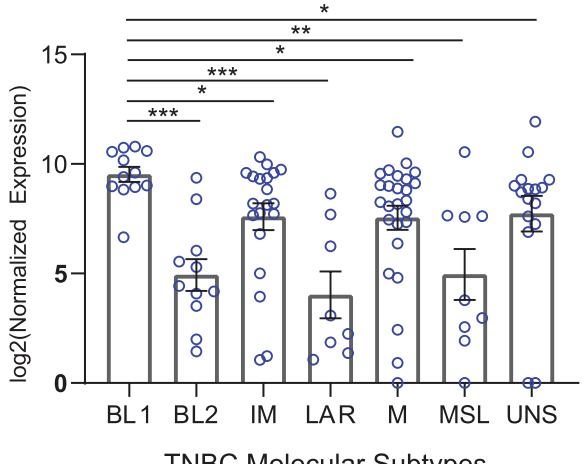

Fig. 4 GSEA analysis of basal-like patients with high $(n=33)$ vs. low $(n=33)$ expression levels of RP11-19E11.1. a Heatmap of top and bottom ranked genes in patients with high and low RP11-19E11.1. b GSEA enrichment results for hallmark repository. c GSEA enrichment results for Oncogenic pathways. d Gene alteration in E2F axis in patients with high $(n=33)$ or low $(n=33)$ RP11-19E11.1 levels or within the basal-like subtype (labeled as basal). e Heatmap of E2F- and E2F-regulated genes in breast cancer subtypes. $\mathbf{f}$ Dot plot showing the expression of RP11-19E11.1 for each patient within the molecular subtypes identified by Vanderbilt classification in 102 TNBC patients from TCGA (Mann-Whitney test). Mean \pm SEM is presented for each condition. ${ }^{*} P<0.05 ;{ }^{* *} p<0.01 ;{ }^{* * *} p<0.001$. HD homodeletion, M mutation, AMP amplification. 


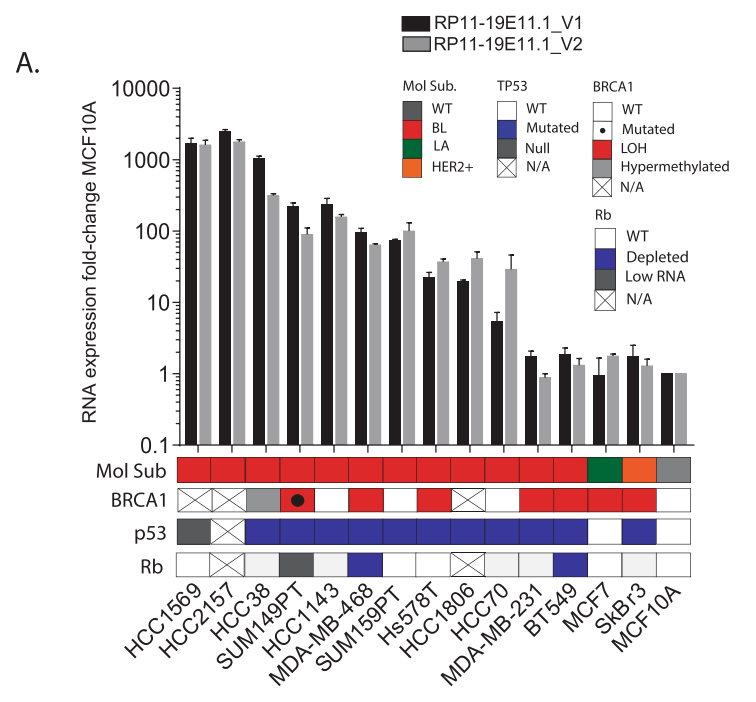

C.

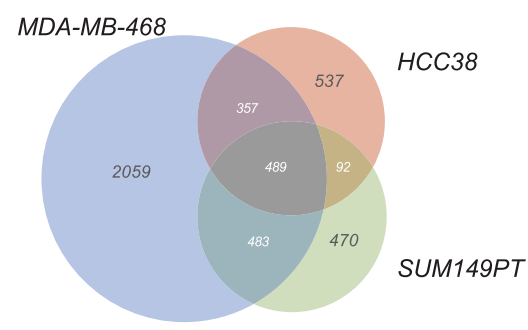

B.
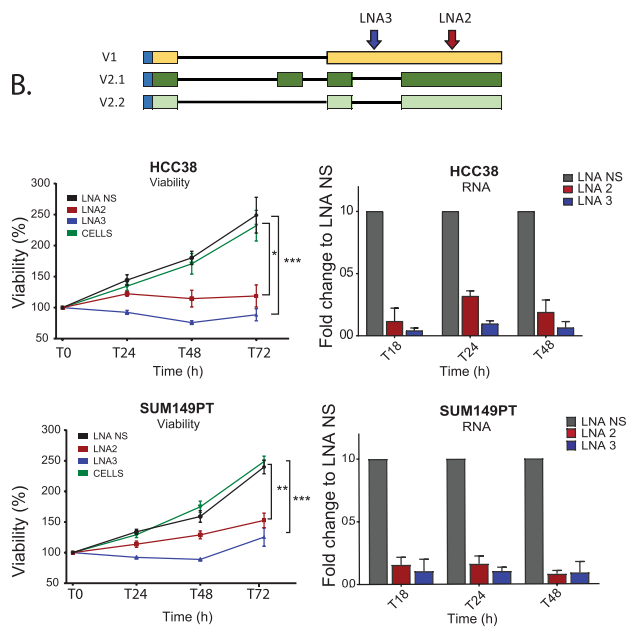

D.
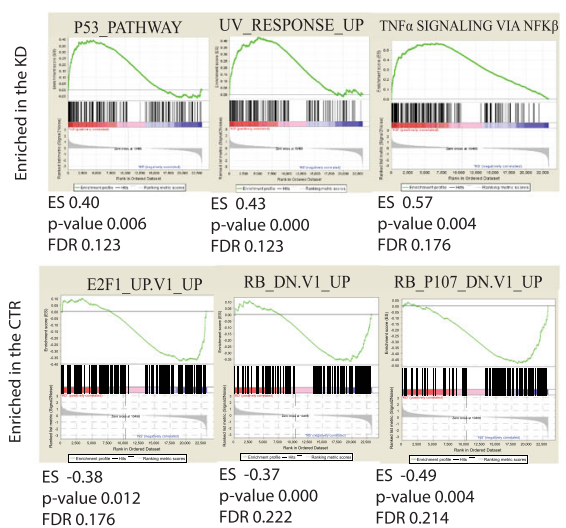

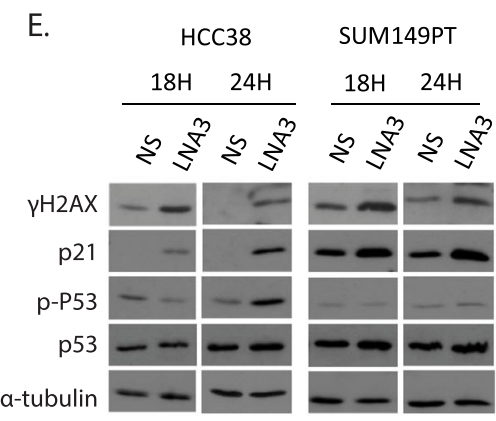

G.
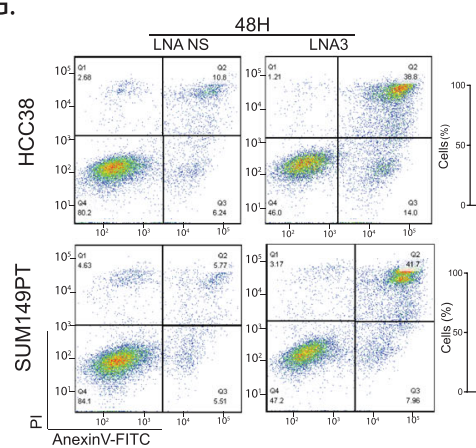

F.

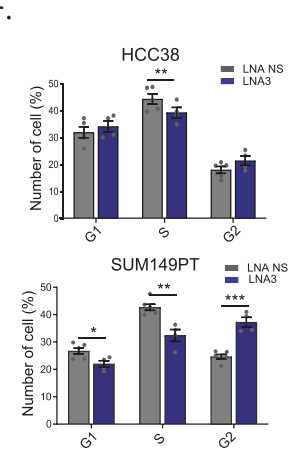

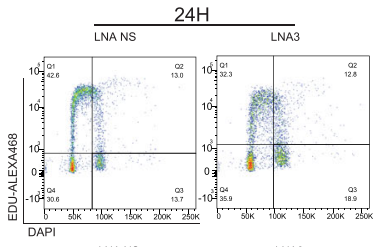
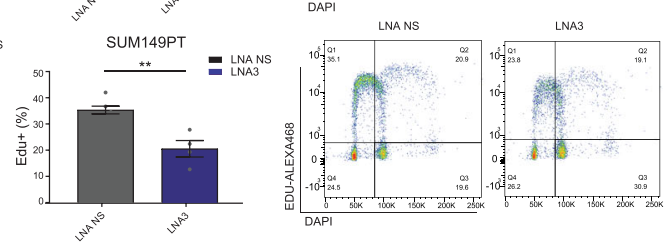

(viability at $72 \mathrm{~h}$ for LNA2 vs. LNA NS $p$ value 0.002 , for LNA2 vs. LNA NS $p$ value $<0.001$ ) (Fig. $5 \mathrm{a}, \mathrm{b}$ ). We found that knock-down was most efficient $18 \mathrm{~h}$ after transfection (>90\% for LNA3, 80\% for LNA2). In addition, we observed that both variants were downregulated after knock-down, irrespective of which LNA was used, suggesting that they could target the unspliced transcript (Supplementary Fig. 4). We next performed RNA-seq after transfection of LNAs targeting RP11-19E11.1 in three cell lines. 489 genes were commonly deregulated in all three cell lines (foldchange $>2$ and false discovery rate $<0.1$, Fig. $5 c$ ). In order to 
Fig. 5 Functional characterization of IncRNA RP11-19E11.1. a RNA levels of both V1 and V2 assessed by qRT-PCR in a panel of basal-like cell lines. MCF10A was used as a reference. b Viability assay (MTT) in HCC38 and SUM149PT cell lines using two different LNAs with distinct target sites (LNA2/LNA3 $25 \mathrm{nM}$ ). c Venn diagram of genes commonly deregulated (fold-change $>2$, false discovery rate (FDR) $<0.1$ ) in the three cell lines after $24 \mathrm{~h}$ of knock-down (LNA1 + LNA2 $50 \mathrm{nM}$ ). d GSEA enrichment results for hallmark and oncogenic pathways after the knock-down of IncRNA RP11-19E11.1. e Protein analysis by WB of $\gamma \mathrm{H} 2 \mathrm{AX}, \mathrm{P} 53, \mathrm{p}-\mathrm{P} 53$, and p21 after 18 and $24 \mathrm{~h}$ after LNA3 transfection (25 nM). f Cell cycle analysis and EdU incorporation analysis (PI-EdU double staining) at $24 \mathrm{~h}$ after LNA3 transfection (25 nM) in HCC 38 and SUM149PT cell lines. Each point represents a single measurement. g Annexin-V analysis after 24 and $48 \mathrm{~h}$ after LNA3 transfection ( $25 \mathrm{nM})$ in HCC38 and SUM149PT cell lines. Experiments were performed at least three times in triplicate. Data are presented as mean \pm SEM. ${ }^{*} P<0.05 ;{ }^{* *} p<0.01 ;{ }^{* * *} p<0.001$ (two-tailed unpaired $t$ test).

identify the main pathways that may be linked to the IncRNA function, we performed GSEA analysis and evaluated enrichment of oncogenic pathways and transcription factor motifs in deregulated genes. Interestingly, our analysis showed that E2F/ $\mathrm{Rb}$ oncogenic pathways and genes enriched for the E2F transcription factor motif were lost after RP11-19E11.1 knockdown (Fig. 5d). In contrast, gene sets that showed upregulation included the p53 pathway, ultraviolet (UV) response, and tumor necrosis factor-a signaling via nuclear factor-KB) (Fig. $5 \mathrm{~d}$ ).

In light of these transcriptomic changes, we analyzed DNA damage markers $(\mathrm{\gamma H} 2 \mathrm{AX})$ and $\mathrm{p} 53$ pathway proteins by western blotting after RP11-19E11.1 knock-down. We observed substantial induction of $\mathrm{\gamma H} 2 \mathrm{AX}$ after $18 \mathrm{~h}$. Also, p53 levels increased after the knock-down, in concert with its canonical target, p21/CDKN1A (Fig. 5e). Upregulation of other canonical p53 target genes, GADD45 and PMAIP1/Noxa, was also observed after knock-down in both cell lines (Supplementary Fig. 5a). Accordingly, cell cycle analysis showed a concomitant decrease in the S-phase fraction in all cell lines (HCC38 $p$ value 0.017 , SUM149PT $p$ value 0.002 ), and a G2/M phase block for SUM149PT cells ( $p$ value $<0.001$ ), confirmed also by DNA synthesis inhibition measured by active 5-ethynyl-2'-deoxyuridine (EdU) incorporation (HCC38 $p$ value 0.0011, SUM149PT $p$ value 0.0014) (Fig. 5f). Given the apparent activation of p53 target genes and the DNA damage response, we asked whether apoptosis could be induced in cells depleted of this IncRNA. Indeed, apoptosis analysis using Annexin-V staining showed induction of cell death within $48 \mathrm{~h}$ of knock-down (Fig. $5 \mathrm{~g}$ ) in both cell lines.

These observations strongly connected our GSEA analysis with phenotypes described after RP11-1E11.1 knock-down, namely, E2F downregulation and cell cycle arrest, followed by activation of apoptotic pathways. We surmise that the resultant apoptosis could be due to replication stress or induction of the DNA damage response after RP11-19E11.1 knock-down.

The induction of p53 downstream genes occurs in a partially p53independent manner

p53 mutations have been reported in $>80 \%$ of triple-negative breast cancers. ${ }^{25}$ Most of the mutations are localized to the DNA binding domain, perturbing the affinity of $\mathrm{p} 53$ for the promoter and altering the expression of transcriptional target genes. ${ }^{26} \mathrm{We}$ noticed that HCC1569, a p53-null cell line, shows high levels of RP11-1911.1, and therefore we asked whether the knock-down of the IncRNA could induce the same response in p53 target genes. Unexpectedly, p21 levels were upregulated independently of p53 in this cell line (Supplementary Fig. 5b, c). Similar results were obtained with the p53-mutated SUM149PT cell line. The depletion of p53 in this cell line did not rescue cell viability after IncRNA knock-down, and levels of p21 increased both at the RNA and protein level independently of p53 protein levels (Supplementary Fig. 5b, d, e). Therefore, apoptosis induction can occur independently of p53 function, which is relevant for a subtype of breast cancer with a high frequency of p53 mutations.

\section{IncRNA RP11-19E11.1 is a E2F1 target gene}

Patients with high levels of expression of RP11-1E11.1 have a specific gene signature related to activation of a subset of E2F target genes. Indeed, the loss of this IncRNA induces cell cycle arrest, thereby reducing proliferation and, subsequently, inducing apoptosis genes. Therefore, we examined three possible scenarios that which could link this IncRNA with the E2F gene signature. First, the IncRNA could control E2F1 transcription. Second, the IncRNA could be regulated by E2F transcription family members and therefore have an indispensable role in maintaining cell proliferation, or third, the IncRNA is involved in regulation of a specific subset of E2F downstream genes. The second and third possibilities are not mutually exclusive.

To evaluate the first option, we checked the levels of the E2F family after RP11-1E11.1 knock-down using the RNA-seq data from three different cell lines. The results did not show significant changes in RNA in any of the E2F family members (Fig. 6a). Protein levels for E2F1 and E2F3 were also evaluated, and no significant changes were observed (Fig. 6b). Therefore, we next evaluated the possibility of the IncRNA being an E2F target gene. Promoter analysis identified several potential E2F motifs around the TSS (transcription starting site) of the IncRNA ( $p$ value $<0.001$, Fig. $6 c$ ). To validate the hypothesis of a direct interaction of E2F1 with the RP11-19E11.1 promoter, we performed chromatin immunoprecipitation (ChIP) analysis. As several putative binding sites were identified, we designed six overlapping primers sets that spanned regions upstream and downstream of the TSS identified by $5^{\prime}$ RACE. Only primer set $4(-141+13$ around TSS) showed significant enrichment compared to the control ( $p$ value 0.003 ) (Fig. $6 \mathrm{~d}$ ). Concordantly, exogenous expression of E2F1 increased the expression of RP1119E11.1 ( $p$ value 0.04 ) to an extent similar to another canonical E2F1 target gene, CCNE2 ( $p$ value 0.017) (Fig. 6e).

Finally, regarding the possibility of RP11-19E11.1 regulating E2F family target genes, we have shown in this study that RP11-19E11.1 depletion is followed by a reversal in the E2F gene signature in vitro, and a robust induction of DNA damage, block of DNA synthesis, and activation of apoptosis pathways. Therefore, we cannot exclude the contribution of an indirect downregulation of some E2F target genes due to a loss of cell fitness after RP11-19E11.1 depletion. Analyses beyond the scope of this report will help define the role of this IncRNA in the regulation of genes controlled directly or indirectly by the E2F transcription factor family.

E2F family members are well-known transcription factors that control several aspects of cell proliferation, from cell cycle progression to DNA damage checkpoints and repair. Several IncRNAs have been identified as E2F targets, including $\mathrm{H} 19,{ }^{27}$ ANRIL, ${ }^{28}$ and ERIC, ${ }^{29}$ and all of them have been shown to exhibit aberrant expression in tumor cells and to play important roles in cancer development. We propose that RP11-19E11.1 may be another member of this group of IncRNAs.

LncRNA RP11-19E11.1 expression has predictive value for drug sensitivity in BL cell lines

Patients with high levels of the IncRNA show specific gene signatures within the BL subtype. We therefore asked whether drug sensitivity within the BL subtype could be predicted by the levels of expression of the IncRNA RP11-19E11.1. To that end, we explored the sensitivity of a selected panel of BL cell lines with different levels of expression of the IncRNA to more than 267 
A.

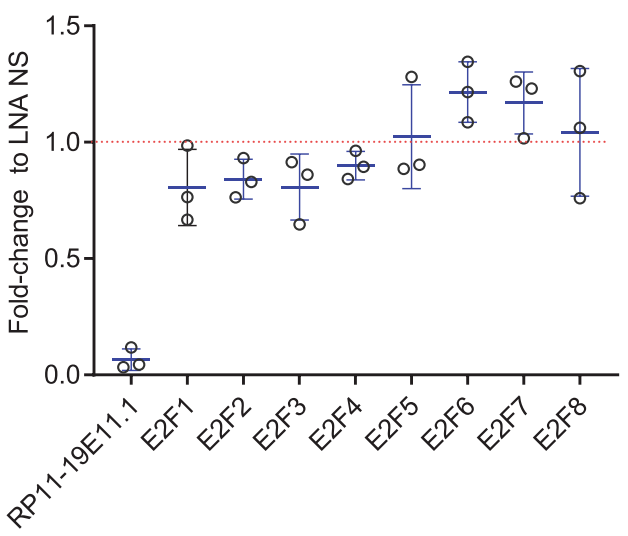

C.

\begin{tabular}{ccccl} 
motif_id & nt from TSS & strand & p-value & matched_sequence \\
\hline \hline MA0024.2 & 251 & + & 0.000133 & GGGGGGAGAGG \\
MA0024.2 & -419 & - & 0.000173 & GGGCCGGGAGC \\
MA0024.2 & 275 & - & 0.000173 & GGGGCCGGAGC \\
MA0024.2 & -374 & - & 0.000185 & GGGGGGCAAAG \\
MA0469.2 & 6 & + & 0.000391 & ACGAGGCGCGCGCTCTG \\
MA0024.2 & 249 & + & 0.000399 & AGGGGGGAGA \\
MA0024.2 & -223 & - & 0.000509 & AGAGAGGGAGG \\
MA0024.2 & -71 & + & 0.000509 & AGAGGGGAGG \\
MA0024.2 & 123 & + & 0.000537 & CGGGGGCCTGC \\
MA0024.3 & 123 & - & 0.000673 & GCAGGCGCCCGG \\
MA0024.2 & -361 & - & 0.000684 & CGCGGGGCAGC \\
MA0024.2 & -471 & - & 0.000711 & CGAGCGGCCGG \\
MA0024.2 & 8 & + & 0.000739 & GAGGCGCGCGC \\
MA0024.2 & 161 & + & 0.000746 & TGCTCGCGAAG \\
MA0469.2 & -166 & - & 0.000853 & GGGAAGGGCGCTCCCCT \\
MA0024.2 & 123 & - & 0.000907 & CAGGCGCCCGG \\
MA0024.3 & -499 & + & 0.000921 & GAGGCCGCCCTT \\
MA0024.1 & 226 & - & 0.000933 & TATCCCGC \\
MA0024.2 & 385 & - & 0.000973 & TGGAGGCAGG \\
MA0024.3 & -163 & - & 0.000982 & AAGGGCGCTCCC \\
MA0471.1 & -411 & - & $3.06 E-05$ & GAGAGGGAGGG \\
MA0470.1 & 252 & + & $9.55 \mathrm{E}-05$ & GGGCGAGAGGA
\end{tabular}

E.
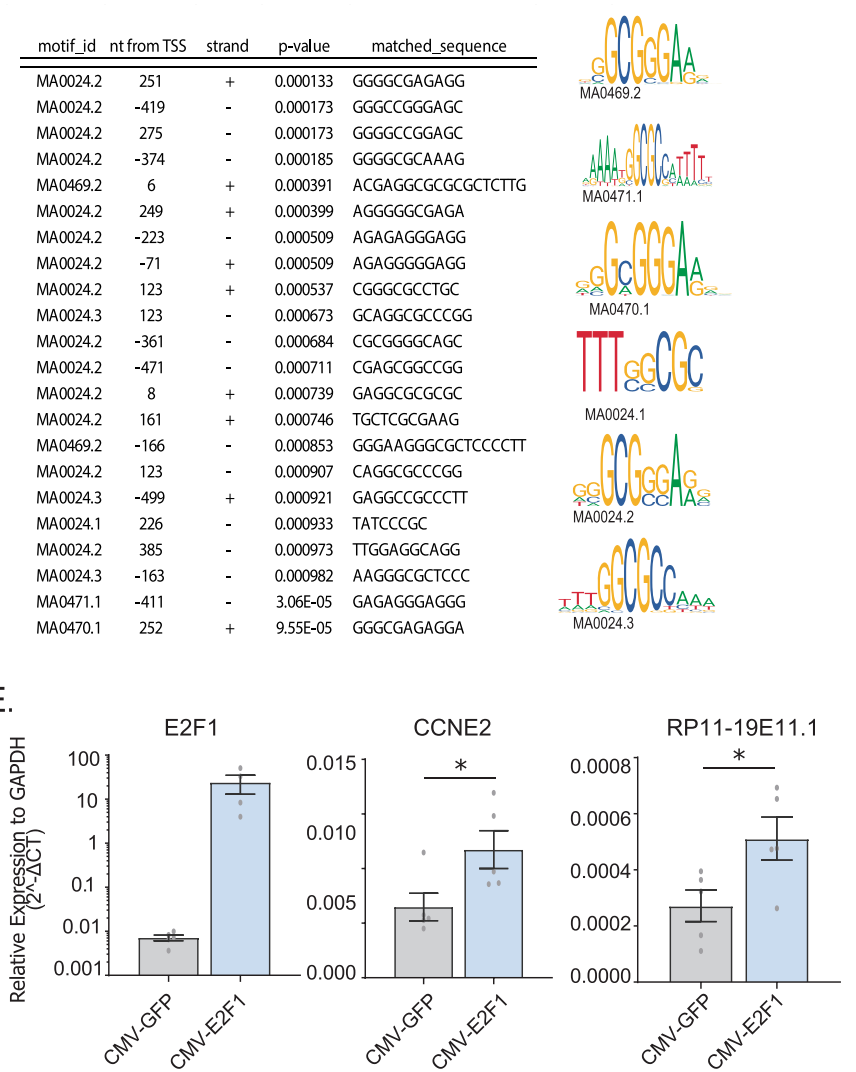

B.

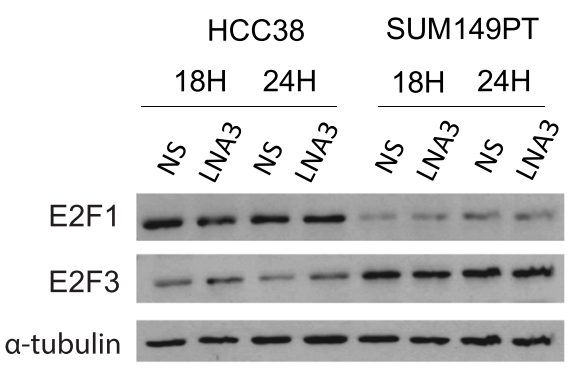

D.
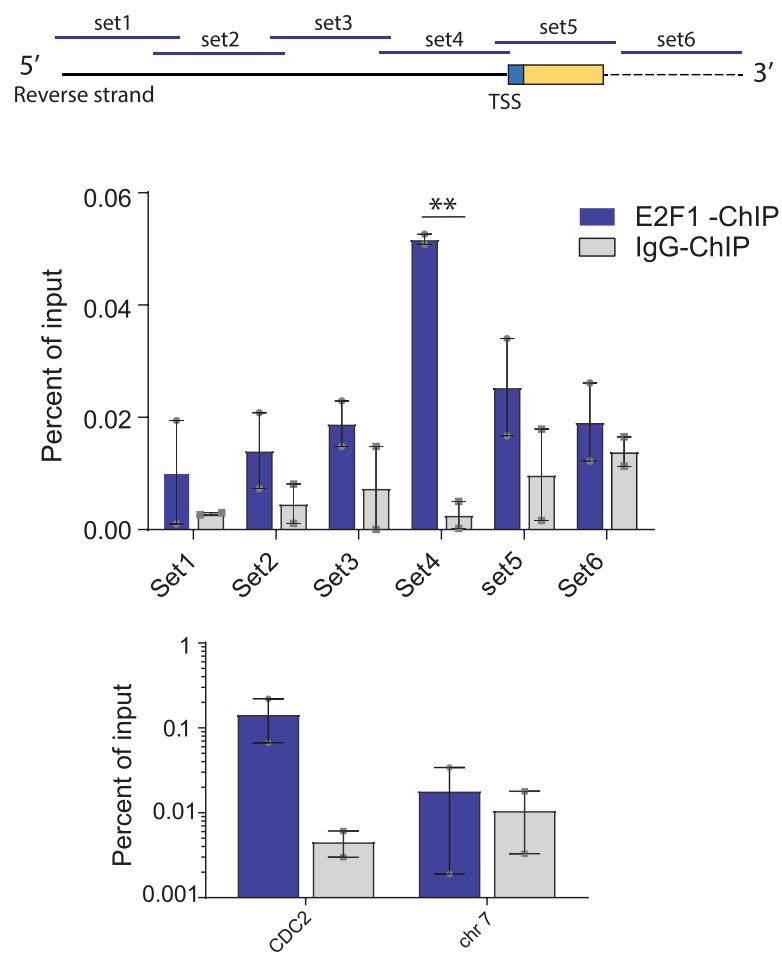

Fig. 6 LncRNA RP11-19E11.1 is an E2F target gene. a RNA expression fold-change of E2F family transcription factors after RP11-19E11.1 knock-down from RNA-seq data. Each point represents the fold-change in each cell line, and for each gene mean value \pm SD is represented. b Western blot for E2F1 and E2F3 after RP11-19E11.1 after 18 and $24 \mathrm{~h}$ using LNA3. c Motif enrichment analysis in the RP11-19E11.1 promoter for E2F transcription factor. d Chromatin immunoprecipitation (ChIP) using E2F1 antibody in HCC38. Two biological replicates are shown (twotailed unpaired $t$ test). e RNA levels of CCNE2 and RP11-19E11.1 in HCC38 and SUM149PT after exogenous overexpression of E2F1 (two-tailed paired $t$ test). Each point represents a single measurement. Data are presented as mean $\pm \mathrm{SEM} .{ }^{*} P<0.05 ;{ }^{* *} p<0.01 ;{ }^{* * *} p<0.001$.

compounds available from The Genomics of Drug Sensitivity in Cancer. ${ }^{30}$ We found drug sensitivity screening data for $14 \mathrm{BL}$ cell lines and overlapped these data with the respective expression levels of the IncRNA obtained from the RNA-seq data available in the CCLE. ${ }^{31}$ We ranked the four top and four bottom cell lines according to the expression of RP11-19E11.1 and compared the average half-maximal inhibitory concentration $\left(\mathrm{IC}_{50}\right)$ of the high expressing cell lines to the average $I_{50}$ of the lowest ones (Fig. 7a). We selected for further analysis those drugs that showed an $\mathrm{IC}_{50}{ }^{\text {high }} / \mathrm{IC}_{50}{ }^{\text {low }}$ ratio $<0.25$, to ensure biological significance in drug sensitivity between groups. For those compounds that showed an $\mathrm{IC}_{50}$ significantly different when comparing the six top/ bottom cell lines according to the IncRNA expression, we analyzed if there was a correlation between drug sensitivity and levels of expression of the IncRNA (Fig. 7b, c). Interestingly, three of the compounds showed a significant negative correlation between expression levels and sensitivity: PAC- 1 ( $p$ value 0.022 ), enzastaurin ( $p$ value 0.023 ), and YM201636 ( $p$ value 0.023 ) (Fig. 7c). PAC-1 is the first pro-caspase activator compound developed and was designated as an orphan drug by the Food Drug Administration in $2016 .^{32}$ Enzastaurin is an inhibitor of the protein kinase C (PKC), which failed the phase III treatment for the treatment of lymphoma. ${ }^{33}$ YM201636, is a PIKfyve (phosphatidylinositol phosphate kinase PIP5KIII) inhibitor, a kinase implicated in Ptdlns(3,5)P2 biosynthesis that regulates a number of intracellular membrane 
A.

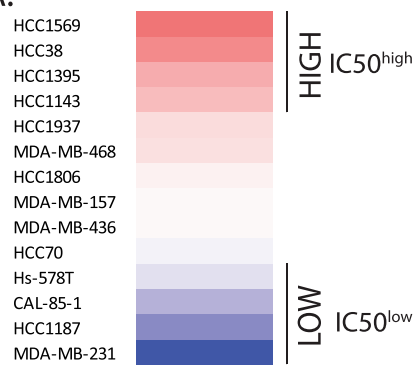

패.

C.

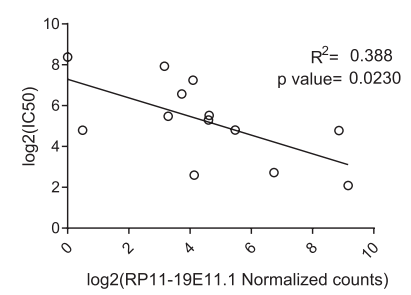

B.

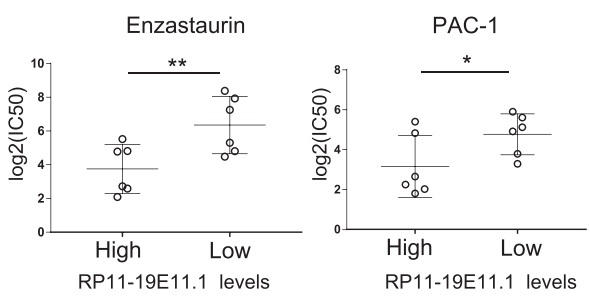

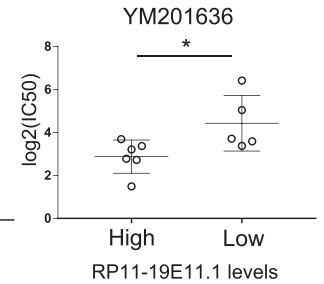

PAC-1

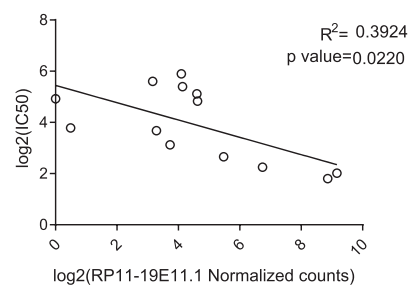

UNC0638

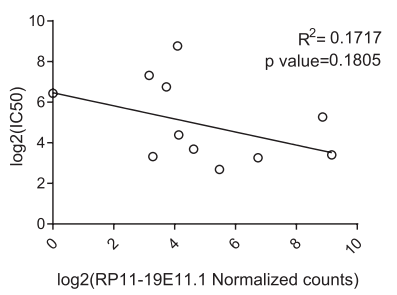

YM201636

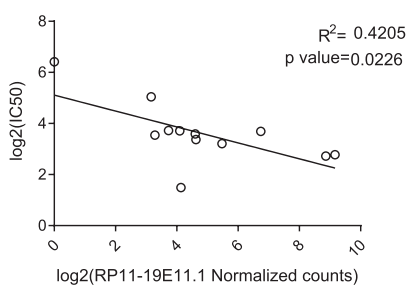

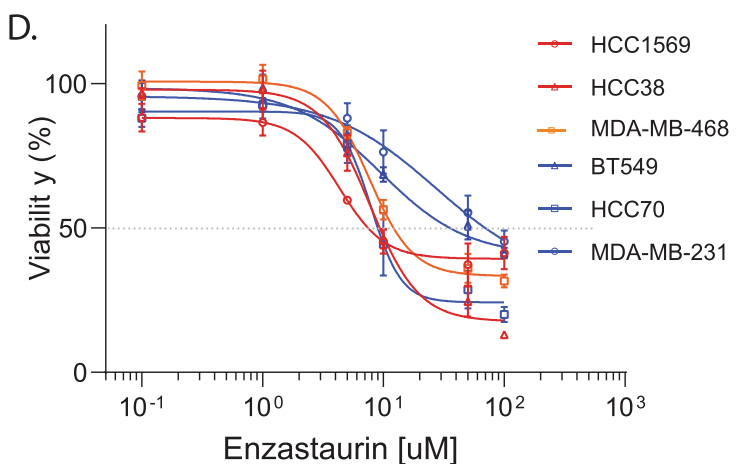
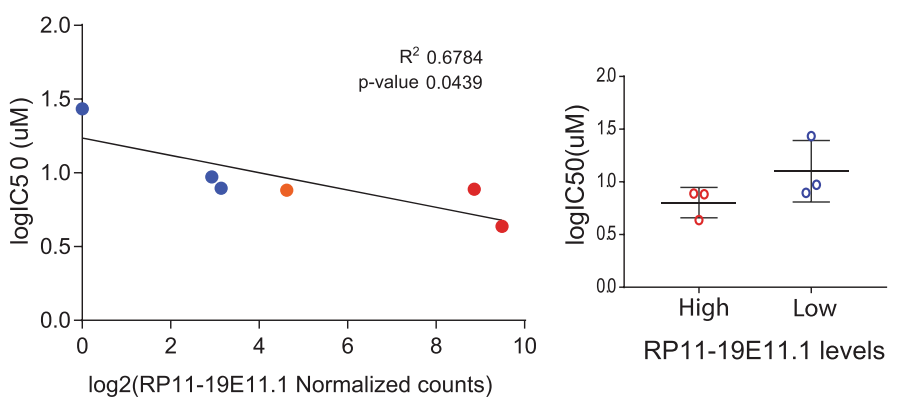

Fig. 7 Drug screening using IncRNA RP11-19E11.1 levels as predictive value. a Basal-like cell lines with drug sensitivity data available ranked according to the expression of RP11-19E11.1 (obtained from CCLE. ${ }^{31}$ ) b Compounds identified with significantly different sensitivity (according to $\mathrm{IC}_{50}$ values) when comparing cell lines with low or high RP11-19E11.1 expressing levels. c Linear correlation between RNA expression and drug sensitivity $\left(\mathrm{IC}_{50}\right)$ for the compounds identified in $\mathbf{b}$. Pearson's correlation test was used. CAL-85-1 cell line is not included (no expression data available). $\mathbf{d}$ Validation of the results for enzastaurin. Data are presented as mean $\pm \mathrm{SEM}$. ${ }^{*} P<0.05 ;{ }^{* *} p<0.01 ;{ }^{* * *} p<0.001$ (unpaired $t$ test unless specified).

trafficking pathways. Its inhibition disrupts endomembrane transport and retroviral release from infected cells. ${ }^{34}$

To validate the compounds identified in this screening, we selected three top/bottom cell lines after ranking our qRT-PCR results for RP11-19E11.1 levels. Time of treatment and viability assessment by metabolic assays were performed using methods similar to those employed in the screening. We found that neither PAC-1 nor YM201636 showed the tendency observed in the screening for the cell lines tested (Supplementary Fig. 6a, b). Enzastaurin, on the other hand, showed the expected results in all cell lines tested, except for SUM149PT (Supplementary Fig. 6c). Therefore, we decided to include another cell line with moderate levels of RP11-19E11.1, MDA-MB-468. Here, we reproduced the results observed in the screening, as a significant negative correlation ( $p$ value 0.044) was observed between RP11-19E11.1 levels and sensitivity to enzastaurin (Fig. $7 d$ ). $I C_{50}$ values showed the same tendency observed in the screening but was not significant.

These results are quite provocative, as PKC activity leads to the activation of several pathways that can modulate a number of cellular processes, including proliferation and anti-apoptotic signaling. ${ }^{35}$ Among others, PKC can activate Raf1 by direct phosphorylation that subsequently can lead to phosphorylation of Rb protein, releasing E2F transcription factors. ${ }^{36}$ In addition, the disruption of Raf1-Rb protein interaction leads to tumor growth and angiogenesis inhibition. ${ }^{37}$ Furthermore, PKC can also activate Ras, which can induce E2F1 expression. ${ }^{38}$ Therefore, PKC inhibition could be an attractive target for further exploration in the treatment of basal breast cancer patients with an E2F activation signature.

\section{DISCUSSION}

Several studies have shown that IncRNA expression can be used to classify breast cancer into subtypes as effectively as PCGs, ${ }^{13,14,39,40}$ providing additional prognostic and predictive value. ${ }^{41}$ We identified a cohort of clinically relevant IncRNAs that are overexpressed in basal-like breast cancer as compared to normal tissue and other breast cancer subtypes, and we suggest that this signature may be useful in future studies aimed at understanding the etiology and treatment of this aggressive tumor.

The consequences and mechanisms of IncRNA dysregulation in basal-like/TNBC subtypes are not well defined. Two studies compared expression levels between TNBC vs. normal tissue using IncRNA microarrays in a small cohort of patients, ${ }^{42,43}$ while another study focused on breast cancer subtypes but did not take into 
account IncRNA expression in normal tissue, ${ }^{40}$ as we have done in our study. Furthermore, our studies are novel because we intersected patient data with cell line RNA-seq data in an effort to use clinical findings in in vitro studies for further functional and mechanistic studies. Interestingly, two of our candidates have been identified as basal-specific IncRNAs in previous studies, the CTD2015G9.2 and RP11-19E11.140,44 Another candidate that we identified is MIR146, the IncRNA host gene for mir-146a, a microRNA extensively studied and shown to play a role in TNBC/basal-like breast cancer. ${ }^{45}$ We validated IncRNA expression using qRT-PCR to confirm the expression pattern reported by RNA-seq in different cell line subtypes. Furthermore, we demonstrated that IncRNA ablation exerted a major impact on cell proliferation and migration/invasion, principal characteristics of the oncogenic signature.

We selected the IncRNA RP11-19E11.1 for further study in detail because its OE correlates with poor prognosis in patients with basal-like breast cancer. Importantly, its depletion impaired cell proliferation and strongly induced apoptosis. While our manuscript was in preparation, another group identified RP11-19E11.1 as a specific basal-like transcript in an independent cohort of tumor samples. ${ }^{44}$ In that study, the authors showed that this IncRNA was regulated through epigenetic modifications, in agreement with our observations, but no detailed description of the transcript, compartment localization, or putative function was provided. Here, we identified and characterized at least three RP11-19E11.1 transcript variants, correcting the actual Gencode annotation. Importantly, we also revealed that the two main IncRNA variants were chromatin-associated. Furthermore, by using GSEA analysis in patients and in cell lines, we linked the IncRNA expression to an E2F signature, demonstrating its connection to E2F experimentally by ChIP. We also provided transcriptome-wide analysis for three different cell lines after RP11-19E11.1 knockdown, further strengthening the connections between ablation of this IncRNA, activation of the DNA damage response, and induction of apoptosis. Therefore, our work has substantially expanded upon previous results, providing a better understanding of the role of RP11-19E11.1 in basal-like breast cancer.

Basal-like breast cancers are generally poorly differentiated tumors, are enriched in embryonic stem cell signatures, and show activation of proliferation-associated factors. ${ }^{46}$ The E2F transcription factors are well-known downstream effectors of the RB tumor suppressor and have a pivotal role in regulating cell cycle progression. ${ }^{47,48}$ They not only transcribe the subset of genes necessary for the transition from G1 to $S$ phase, but they also have a role in controlling mitosis, DNA damage checkpoints and repair, and apoptosis. ${ }^{47,48}$ E2Fs are known to affect cancer development, ${ }^{22}$ and $\mathrm{Rb}$ dysfunction in TNBC is estimated to occur in $~ 30 \%$ of the cases. ${ }^{49}$ The influence of the E2F transcription factors in breast cancer development is undisputed. ${ }^{23}$ In this study, we identified an E2F oncogenic signature in patients with upregulated RP11-19E11.1 levels. Furthermore, our results showed RP11-19E11.1 to be an E2F1 target and a chromatin-associated IncRNA. Indeed, knock-down of RP11-19E11.1 induced robust inhibition of DNA synthesis and reduced expression of E2F target genes, which was followed by a strong activation of DNA damage response and apoptotic pathways. We showed that these effects could occur independently of p53. Altogether, these results suggest that RP11-19E11.1 function could be linked to that of E2F. Several examples of IncRNAs controlling cell cycle can be found in the literature. ${ }^{50}$ For instance, ANRIL, an E2F1 target gene, promotes gene silencing by recruiting repressors to the INK4 promoter. Another E2F1 target gene, H19/miR-67, seems to be involved in pRB pathway inactivation. Therefore, in order to elucidate the possible scenarios in which RP11-19E11.1 could be involved in regulating cell cycle, future studies should investigate RNA-DNA and RNA-protein interactions.

Patients with elevated RP11-19E11.1 expression shared a specific oncogenic signature linked to poor prognosis. We speculated that these patients would also show different sensitivities to drug treatment. Taking advantage of drug screening published data in cell lines, we identified a PKC inhibitor as a potential compound for further study. Notably, PKC not only shares a connection to E2F activation but is also involved in key steps of viral replication. ${ }^{51}$ The idea of virus-initiated breast cancer has been proposed but is controversial. More compellingly, a recently published study found that the expression of human endogenous retrovirus- $K$ is strongly associated with the basal-like breast cancer, and a strong association with RB phosphorylation and cell cycle activation was observed in these patients. ${ }^{52}$

In conclusion, this study identifies clinically relevant basal-like IncRNAs using a large cohort of samples from TCGA. Some of the candidates identified may have prognostic value and may be directly implicated in the oncogenic phenotype. Finally, we characterized the IncRNA, RP11-19E11.1, identifying a novel chromatin-associated and E2F target IncRNA. Our results show that this IncRNA is necessary for cell cycle progression, and its ablation impairs cell viability by inducing apoptosis. We also identified an E2F-specific signature linked to the expression of RP11-19E11.1, and have used cell lines expressing this IncRNA to identify possible drug therapies, which could be tested in TNBC patients.

\section{METHODS}

\section{TCGA data}

The read count tables for all the sequencing samples $(n=1162)$ in TCGA were downloaded from The National Cancer Institute's Genomic Data Commons (GDC). The count tables were normalized based on their library size factors using DEseq2, and differential expression analysis was performed. Clustering was performed using iCellR (https://github.com/ rezakj/iCellR) by selecting the dispersed genes running principal component analysis and t-SNE. All the methylation $\beta$ values for the samples with Illumina 450 methylation array data $(n=892)$ were downloaded from GDC.

\section{RNA-seq data processing}

All the raw sequencing reads from our samples (Gene Expression Omnibus (GEO) https://identifiers.org/geo:GSE138606 (2019)) and the cell lines downloaded from the GEO repository, https://identifiers.org/geo:GSE73526 (2016) and https://identifiers.org/geo:GSE48213 (2013), were mapped to the human reference genome (GRCh37/hg19) using the STAR aligner (v2.5.0c $)^{54}$. Alignments were guided by a Gene Transfer Format file. The mean read insert sizes and their standard deviations were calculated using Picard tools (v.1.126) (http://broadinstitute.github.io/picard). The read count tables were generated using HTSeq $(v \cdot 0.6 .0)^{55}$ normalized based on their library size factors using DEseq $2^{56}$ and differential expression analysis was performed. The read per million normalized BigWig files were generated using BEDTools $(v \text {.2.17.0) })^{57}$ and bedGraphToBigWig tool (v.4). All downstream statistical analyses and generating plots were performed in $\mathrm{R}$ environment (v.3.1.1) (http://www.r-project.org/).

\section{Cell culture}

Breast cancer cells MDA-MB-231, Hs578T, MDA-MB-157, MDA-MB-468, MCF7, SkBr3, and BT474 were cultured in Dulbecco's modified Eagle's mMedium: Nutrient Mixture F12 (DMEM/F12) (Corning) supplemented with $10 \%$ fetal bovine serum (FBS), P/S (50 U/mL), and L-Glut (1\%). SUM149PT and SUM159PT were cultured with DMEM/F12 supplemented with $5 \% \mathrm{FBS}$, $\mathrm{P} / \mathrm{S}(50 \mathrm{U} / \mathrm{mL})$, and L-Glut $(1 \%)$, insulin $(5 \mu \mathrm{g} / \mathrm{mL})$, and hydrocortisone $(1 \mu \mathrm{g} /$ $\mathrm{mL}$ ). Breast cancer cells HCC2157, HCC38, HCC2157, BT549, HCC70, HCC1143, and T-47D were cultured in RPMI medium supplemented with $10 \% \mathrm{FBS}, \mathrm{P} / \mathrm{S}(50 \mathrm{U} / \mathrm{mL})$, and L-Glut $(1 \%)$. Normal breast MCF10A cells were cultured in DMEM/F12 (Corning) medium supplemented with $5 \%$ horse serum, epidermal growth factor $(20 \mathrm{ng} / \mathrm{mL})$, cholera toxin $(100 \mathrm{ng} / \mathrm{mL})$, insulin $(0.01 \mathrm{mg} / \mathrm{mL})$, and hydrocortisone $(500 \mathrm{ng} / \mathrm{mL})$. All cells were cultured at $37{ }^{\circ} \mathrm{C}$ with $5 \% \mathrm{CO}_{2}$. All cell lines were obtained from ATCC, except from SUM149PT and SUM159PT (gifted from Neel BG laboratory).

To ensure mycoplasma-free culture, cells were tested periodically using the Universal Mycoplasma Detection Kit (ATCC, 30-1012K). 
RNA interference (siRNA and LNA) Protocol

siRNA at $20 \mathrm{nM}$ (Dharmacon) or Antisense LNA GapmeRs at 25/50 nM (Qiagen) transfection mix was prepared in OptiMEM (Gibco) and RNAiMAX at $2.5 \mu \mathrm{L} / \mathrm{mL}$ (Invitrogen) for $10 \mathrm{~min}$ at room temperature. Transfection mix was then added to the cells at the time of seeding. After $6 \mathrm{~h}$, the media were replaced for fresh new media. siRNA and LNAs sequences are in Supplementary Methods.

\section{Cell transfection protocol}

Cells were seeded in 6-well plates at a confluence of $20-30 \%$. Next day, transfection mix was prepared using FuGene HD reagent (Promega) at 3:1 ratio with $1 \mu \mathrm{g}$ of pCMV-E2F1 or pCDH-EGFP in OptiMEM. After $10 \mathrm{~min}$, the mixture was added to the cells with a final volume of $1 \mathrm{~mL}$ in $0.5 \%$ DMEM/ F12 media. After 6 h, one volume of 10\% FBS media was added. Next day, transfection media was replaced for fresh media and cells were collected for analysis after 48 or $72 \mathrm{~h}$.

\section{Cell fractionation}

Nuclear/cytoplasmic fractionation was performed as described..$^{53}$ Briefly, cells were rinsed three times with cold phosphate-buffered saline (PBS) and scraped carefully. After spinning, disruption buffer $\left(\mathrm{KCl} 10 \mathrm{mM}, \mathrm{MgCl}_{2}\right.$ $1.5 \mathrm{mM}$, Tris-Cl, pH 7.5, $20 \mathrm{mM}$, and dithiothreitol (DTT) $1 \mathrm{mM}$ ) was added and let to stand for $10 \mathrm{~min}$. A type B Dounce was used to disrupt the cytoplasm using 10-15 strokes. When $~ 90 \%$ of the cytoplasm was broken, $0.1 \%$ of Triton $X$ was added, mixed by inversion five times, and centrifuged for $5 \mathrm{~min}$ at 1500 r.p.m. The supernatant containing the cytoplasmic fraction was recovered by this method. Both supernatant and nuclei (pellet) were processed for RNA extraction using the SurePrep Nuclear or Cytoplasmic RNA Purification Kit (Fisher, BP2805-50) following user manual instructions. DNAasel treatment was performed for both fractions (Norgen Biotek cat. num. 25710).

\section{Chromatin precipitation}

Cell fractionation was performed as described above. The pellet containing the nuclear fraction was then resuspended in $125 \mu \mathrm{L}$ of cold NUN1 buffer ( $20 \mathrm{mM}$ Tris- $\mathrm{HCl}, 75 \mathrm{mM} \mathrm{NaCl}, 0.5 \mathrm{mM}$ EDTA, 50\% glycerol) and transferred to a new Eppendorf. Then, $1.2 \mathrm{~mL}$ of cold NUN2 buffer (20 mM HEPES-KOH, pH 7.6, 300 mM NaCl, 0.2 mM EDTA, $7.5 \mathrm{mM} \mathrm{MgCl}_{2}$, $1 \% \mathrm{NP} 40,1 \mathrm{M}$ urea) was added and vortexed. Samples were kept on ice for $15 \mathrm{~min}$, vortexing every 3-4 min. Chromatin was recovered by centrifuging at $16,000 \times g$ for $10 \mathrm{~min}$ at $4{ }^{\circ} \mathrm{C}$. RNA was extracted from the supernatant fraction using TRIzol LS (Invitrogen) and for the chromatin fraction with TRIzol (Invitrogen).

Reverse transcription and quantitative real-time PCR

Total RNA was isolated using the TRlzol reagent (Fisher). RNA was quantified with NanoDrop (Thermo Scientific) and RNA was reverse transcribed using Verso cDNA Synthesis Kit (Thermo Scientific). Quantitative reverse transcription PCR was performed using CFX96 Touch Real-Time PCR Detection System (Bio-Rad). Relative quantity of expression was calculated with the $\triangle \triangle \mathrm{Ct}$ method using GAPDH as an internal control. Primer sequences are in Supplementary Methods.

\section{Protein extraction and Western blot}

Cells were trypsinized and washed with PBS. The pellet was then resuspended in fresh ELB buffer (50 mM HEPES, pH 7, $150 \mathrm{mM} \mathrm{NaCl}, 5 \mathrm{mM}$ EDTA, $0.1 \%$ NP40, $10 \%$ glycerol) supplemented with a protease and phosphatase inhibitor cocktail (1 mM DTT, $0.5 \mathrm{mM}$ AEBSF (4-(2-aminoethyl) benzenesulfonyl fluoride hydrochloride), $2 \mu \mathrm{g} / \mathrm{mL}$ leupeptin, $2 \mu \mathrm{g} / \mathrm{mL}$ aprotinin, $10 \mathrm{mM} \mathrm{NaF}, 50 \mathrm{mM} \beta$-glycerophosphate). Twenty micrograms of protein was loaded in a $10-15 \%$ acrylamide gels. Primary antibodies were diluted in 3\% bovine serum albumin TBS-T (Tris-buffered saline with Tween20) and incubated $\mathrm{O} / \mathrm{N}$ at $4{ }^{\circ} \mathrm{C}$. The primary antibody used and dilutions are a-tubulin (Sigma T5168, 1:5000), E2F1 (CST \#3742, 1:1000), E2F3 (GeneTex GTX11843, 1:2000), p21 (CST \#2947, 1:1000), P53 (Santa Cruz FL-393, 1:1000), p-P53 (CST \#9286, 1:1000), үH2AX (CST \#9718, 1:1000). All blots were derived from the same experiment and were processed in parallel. Un-cropped blots can be found in the Supplementary information file.

\section{FACS analysis}

For EdU and cell cycle analysis, Click-iT EdU Alexa Fluor 488 Kit was used (Invitrogen C10337) following the manufacturer's instructions.

Annexin- $V$ staining was performed using Annexin-V Kit (Mileny Biotec, 130-092-052) following the manufacturer's instructions. All analyses were performed with LSRII UV cell analyzer (BD Bioscience) and FlowJo software.

\section{Viability assays and compounds}

For drug sensitivity evaluation, cells were seeded in a 96-well plate at 4000 cells/well. The next day, cells were incubated at $37^{\circ} \mathrm{C}$ with different concentrations of the test compounds. Control cultures were incubated with dimethyl sulfoxide (DMSO). After 48 or $72 \mathrm{~h}$, media were replaced with fresh media supplemented with MTT (Sigma) at a final concentration of $0.5 \mathrm{mg} / \mathrm{mL}$. After $1.5 \mathrm{~h}$, media were removed and formazan crystals were dissolved with $100 \mu \mathrm{l}$ of DMSO. Absorbance at $570 \mathrm{nM}$ was read in the spectrophotometer (TECAN Infinite M200). The drugs used in this assay were PAC-1 (S2738, Selleckchem), YM201636 (ref. 13576, Cayman Chemical), and enzastaurin (ref. HY-10342, MedChemExpress).

For viability assays after RNA interference, cells were seeded at 4000 cells/well and transfected at the same time as explained in the previous section. After $6 \mathrm{~h}$, media were replaced with fresh new media. MTT assay was performed as explained above each day and viability was compared to T0. All experiments were repeated at least three times with different cell passage number, with three replicates per experiment.

\section{Rapid amplification of cDNA ends}

Nuclear RNA was isolated from HCC2157 cells lines following the protocol described above. RACE was performed using the SMARTer RACE $5^{\prime} / 3^{\prime}$ Kit (Clontech) as per the manufacturer's instructions. Primers were designed at known regions of the transcripts in order to achieve accurate $5^{\prime}$ or $3^{\prime}$ ends (Supplementary Methods).

\section{Chromatin immunoprecipitation}

ChIP was performed following the previous described method with some modifications ${ }^{58}$. Nuclei were obtained from $1 \times 10^{7}$ HCC38 cells and crosslinked with $1 \%$ formaldehyde. Sonication was performed with Branson Sonifier 450 on ice to obtain an average DNA length of 400-600 bp. The equivalent of $25 \mu \mathrm{g}$ DNA was used per ChIP reaction. Chromatin was precleared with protein $A$ and then incubated with $2 \mu \mathrm{g}$ of each antibody, E2F1 and lgG (Millipore \#17-10061 Crosslinks) O/N. Chromatin was resuspended in $200 \mu \mathrm{L}$ of $10 \mathrm{mM}$ Tris, $\mathrm{pH} 8.0$ buffer, and enrichment was assayed by quantitative PCR. Primers used are described in Supplementary Methods.

\section{Reporting summary}

Further information on research design is available in the Nature Research Reporting Summary linked to this article.

\section{DATA AVAILABILITY}

The raw RNA-sequencing data (supporting Fig. 1) used during the study are publicly available in the NCBI Gene Expression Omnibus (GEO) repository: https://identifiers. org/geo:GSE73526 and https://identifiers.org/geo:GSE48213. The read count tables (Supporting Figs $1-4)$ for all the sequencing samples $(n=1162)$ in TCGA are publicly available at The National Cancer Institute's (NCI) Genomic Data Commons (GDC) (https://portal.gdc.cancer.gov/). RNA-sequencing datasets (Supporting Figs 3-6) generated during the current study are publicly available in the GEO repository: https://identifiers.org/geo:GSE138606. The Drug sensitivity in Breast Cancer Cell lines data (Supporting Fig. 7) are publicly available at https://www.cancerrxgene.org/ downloads/drug_data?tissue=BRCA. Additional datasets used and generated during the study will be made available on request from the corresponding author, Prof. Francisco J. Esteva, as described in the following metadata record: https://doi.org/ 10.6084/m9.figshare.10266527 $7^{59}$.

\section{CODE AVAILABILITY}

The code used in the present study is available at https://github.com/rezakj/iCellR. 
Received: 17 July 2019; Accepted: 20 November 2019; Published online: 06 January 2020

\section{REFERENCES}

1. Siegel, R. L., Miller, K. D. \& Jemal, A. Cancer statistics, 2019. CA Cancer J. Clin. 69 7-34 (2019).

2. Perou, C. M. et al. Molecular portraits of human breast tumours. Nature 406, 747 (2000).

3. Sørlie, T. et al. Gene expression patterns of breast carcinomas distinguish tumor subclasses with clinical implications. Proc. Natl Acad. Sci. USA 98, 10869-10874 (2001)

4. Carey, L. A. et al. The triple negative paradox: primary tumor chemosensitivity of breast cancer subtypes. Clin. Cancer Res. 13, 2329-2334 (2007).

5. Prat, A. \& Perou, C. M. Deconstructing the molecular portraits of breast cancer. Mol. Oncol. 5, 5-23 (2011).

6. Lehmann, B. D. et al. Identification of human triple-negative breast cancer subtypes and preclinical models for selection of targeted therapies. J. Clin. Invest. 121, 2750-2767 (2011).

7. Carninci, P. et al. The transcriptional landscape of the mammalian genome. Science 309, 1559-1563 (2005).

8. Prensner, J. R. \& Chinnaiyan, A. M. The emergence of IncRNAs in cancer biology. Cancer Discov. 1, 391-407 (2011).

9. Ulitsky, I. \& Bartel, D. P. lincRNAs: genomics, evolution, and mechanisms. Cell 154, 26-46 (2013).

10. Mattick, J. S. Non-coding RNAs: the architects of eukaryotic complexity. EMBO Rep. 2, 986-991 (2001).

11. Freedman, M. L. et al. Principles for the post-GWAS functional characterization of cancer risk loci. Nat. Genet. 43, 513-518 (2011).

12. Quinn, J. J. \& Chang, H. Y. Unique features of long non-coding RNA biogenesis and function. Nat. Rev. Genet. 17, 47 (2016).

13. Su, X. et al. Comprehensive analysis of long non-coding RNAs in human breast cancer clinical subtypes. Oncotarget 5, 9864-9876 (2014).

14. Liu, Y. R. et al. Comprehensive transcriptome analysis identifies novel molecular subtypes and subtype-specific RNAs of triple-negative breast cancer. Breast Cancer Res. 18, 33 (2016).

15. Liu, $\mathrm{H}$. et al. Long non-coding RNAs as prognostic markers in human breast cancer. Oncotarget 7, 20584 (2016).

16. Ciriello, G. et al. Comprehensive molecular portraits of invasive lobular breast Cancer Cell 163, 506-519 (2015)

17. Van Grembergen, O. et al. Portraying breast cancers with long noncoding RNAs. Sci. Adv. 2, e1600220 (2016)

18. Sun, Q., Hao, Q. \& Prasanth, K. V. Nuclear long noncoding RNAs: key regulators of gene expression. Trends Genet. 34, 142-157 (2018).

19. Werner, M. S. \& Ruthenburg, A. J. Nuclear fractionation reveals thousands of chromatin-tethered noncoding RNAs adjacent to active genes. Cell Rep. 12, 1089-1098 (2015).

20. Beltran, A. S., Graves, L. M. \& Blancafort, P. Novel role of Engrailed 1 as a prosurvival transcription factor in basal-like breast cancer and engineering of interference peptides block its oncogenic function. Oncogene 33, 4767-4777 (2014)

21. Jones, P. A. \& Baylin, S. B. The fundamental role of epigenetic events in cancer Nat. Rev. Genet. 3, 415-428 (2002).

22. Nevins, J. R. The Rb/E2F pathway and cancer. Hum. Mol. Genet. 10, 699-703 (2001).

23. Sun, C. C. et al. Comprehensive analysis of the expression and prognosis for E2Fs in human breast cancer. Mol. Ther. https://doi.org/10.1016/j.ymthe.2019.03.019 (2019).

24. Chen, X. et al. TNBCtype: a subtyping tool for triple-negative breast cancer. Cancer Inf. 11, 147-156 (2012).

25. Cancer Genome Atlas, N. Comprehensive molecular portraits of human breast tumours. Nature 490, 61-70 (2012).

26. Muller, P. A. \& Vousden, K. H. p53 mutations in cancer. Nat. Cell Biol. 15, 2-8 (2013).

27. Berteaux, N. et al. H19 mRNA-like noncoding RNA promotes breast cancer cell proliferation through positive control by E2F1. J. Biol. Chem. 280, 29625-29636 (2005).

28. Wan, G. et al. Long non-coding RNA ANRIL (CDKN2B-AS) is induced by the ATME2F1 signaling pathway. Cell Signal. 25, 1086-1095 (2013).

29. Feldstein, O. et al. The long non-coding RNA ERIC is regulated by E2F and modulates the cellular response to DNA damage. Mol. Cancer 12, 131 (2013).

30. Yang, W. et al. Genomics of drug sensitivity in cancer (GDSC): a resource for therapeutic biomarker discovery in cancer cells. Nucleic Acids Res. 41, D955-D961 (2013)
31. Barretina, J. et al. The Cancer Cell Line Encyclopedia enables predictive modelling of anticancer drug sensitivity. Nature 483, 603-607 (2012).

32. Putt, K. S. et al. Small-molecule activation of procaspase-3 to caspase-3 as a personalized anticancer strategy. Nat. Chem. Biol. 2, 543-550 (2006).

33. Crump, M. et al. Randomized, double-blind, phase III trial of enzastaurin versus placebo in patients achieving remission after first-line therapy for high-risk diffuse large B-cell lymphoma. J. Clin. Oncol. 34, 2484-2492 (2016).

34. Jefferies, H. B. J. et al. A selective PIKfyve inhibitor blocks Ptdlns $(3,5)$ P(2) production and disrupts endomembrane transport and retroviral budding. $E M B O$ Rep. 9, 164-170 (2008).

35. McCubrey, J. A. et al. Roles of the Raf/MEK/ERK pathway in cell growth, malignant transformation and drug resistance. Biochim. Biophys. Acta 1773, 1263-1284 2007).

36. Wang, S., Ghosh, R. N. \& Chellappan, S. P. Raf-1 physically interacts with Rb and regulates its function: a link between mitogenic signaling and cell cycle regulation. Mol. Cell. Biol. 18, 7487-7498 (1998).

37. Dasgupta, P. et al. Disruption of the Rb-Raf-1 interaction inhibits tumor growth and angiogenesis. Mol. Cell. Biol. 24, 9527-9541 (2004).

38. Berkovich, E. \& Ginsberg, D. Ras induces elevation of E2F-1 mRNA levels. J. Biol. Chem. 276, 42851-42856 (2001).

39. Yan, X. et al. Comprehensive genomic characterization of long non-coding RNAs across human cancers. Cancer Cell 28, 529-540 (2015).

40. Bradford, J. R., Cox, A., Bernard, P. \& Camp, N. J. Consensus analysis of whole transcriptome profiles from two breast cancer patient cohorts reveals long noncoding RNAs associated with intrinsic subtype and the tumour microenvironment. PLOS ONE 11, e0163238 (2016).

41. Jiang, Y. Z. et al. Transcriptome analysis of triple-negative breast cancer reveals an integrated mRNA-IncRNA signature with predictive and prognostic value. Cancer Res 76, 2105-2114 (2016).

42. Chen, C. et al. Microarray expression profiling of dysregulated long non-coding RNAs in triple-negative breast cancer. Cancer Biol. Ther. 16, 856-865 (2015).

43. Shen, $X$. et al. Identification of novel long non-coding RNAs in triple-negative breast cancer. Oncotarget 6, 21730-21739 (2015).

44. Han, Y. J. et al. LncRNA BLAT1 is upregulated in basal-like breast cancer through epigenetic modifications. Sci. Rep. 8, 15572 (2018).

45. Sandhu, R. et al. Overexpression of miR-146a in basal-like breast cancer cells confers enhanced tumorigenic potential in association with altered p53 status. Carcinogenesis 35, 2567-2575 (2014).

46. Ben-Porath, I. et al. An embryonic stem cell-like gene expression signature in poorly differentiated aggressive human tumors. Nat. Genet 40, 499-507 (2008).

47. Ren, B. et al. E2F integrates cell cycle progression with DNA repair, replication and G(2)/M checkpoints. Genes Dev. 16, 245-256 (2002).

48. Chen, H. Z., Tsai, S. Y. \& Leone, G. Emerging roles of E2Fs in cancer: an exit from cell cycle control. Nat. Rev. Cancer 9, 785-797 (2009).

49. Witkiewicz, A. K. \& Knudsen, E. S. Retinoblastoma tumor suppressor pathway in breast cancer: prognosis, precision medicine, and therapeutic interventions. Breast Cancer Res 16, 207 (2014).

50. Kitagawa, M., Kitagawa, K., Kotake, Y., Niida, H. \& Ohhata, T. Cell cycle regulation by long non-coding RNAs. Cell Mol. Life Sci. 70, 4785-4794 (2013).

51. Mondal, A. et al. Influenza virus recruits host protein kinase $C$ to control assembly and activity of its replication machinery. Elife 6, https://doi.org/10.7554/ elife.26910 (2017).

52. Johanning, G. L. et al. Expression of human endogenous retrovirus-K is strongly associated with the basal-like breast cancer phenotype. Sci. Rep. 7, 41960 (2017).

53. Rio, D. C., Ares, M. Jr., Hannon, G. J. \& Nilsen, T. W. Preparation of cytoplasmic and nuclear RNA from tissue culture cells. Cold Spring Harb. Protoc. 2010, pdb prot5441 (2010).

54. Dobin, A. et al. STAR: ultrafast universal RNA-seq aligner. Bioinformatics 29, 15-21 (2013).

55. Anders, S. et al. HTSeq-a Python framework to work with high-throughput sequencing data. Bioinformatics 31, 166-169 (2015).

56. Love, M. I. et al. Moderated estimation of fold change and dispersion for RNA-seq data with DESeq2. Genome Biology 15 (2014).

57. Quinlan, A. R. et al. BEDTools: a flexible suite of utilities for comparing genomic features. Bioinformatics 26, 841-842 (2010).

58. Orlando, V. Analysis of Chromatin Structure byin VivoFormaldehyde CrossLinking. Methods 11, 205-214 (1997).

59. Giro-Perafita, A., Luo, L., Khodadadi-Jamayran, A., Thompson, M., Akgol Oksuz, B., Tsirigos, A. et al. Metadata supporting data files in the published article: LncRNA RP11-19E11 is an E2F1 target required for proliferation and survival of basal breast cancer. figshare. Online resource. https://doi.org/10.6084/m9. figshare.10266527 (2019). 


\section{ACKNOWLEDGEMENTS}

This research was supported by The Breast Cancer Research Foundation (BCRF) and Shifrin-Myers Fund awarded to F.J.E. and by NIH grant 5R21CA220518 awarded to I.S.

\section{AUTHOR CONTRIBUTIONS}

Conception and design A.G.P., B.D.D., I.S., and F.J.E. Design and development of the pipeline: A.K.-J., B.A.-O., A.T. Development of methodology: A.G.-P., L.L. Bioinformatic data analysis: A.G.-P. and A.K-J.J. Technical assistance: M.T. Analysis and interpretation of data: A.G.-P., A.K.-J., A.T., B.D.D., I.S., and F.J.E. Writing first draft: A.G.-P. Reviewing and revising the manuscript: A.G.-P., B.D.D., I.S., and F.J.E. Study supervision: A.T., B.D.

D., I.S., and F.J.E. All the authors read and approved the manuscript.

\section{COMPETING INTERESTS}

The authors declare no competing interests.

\section{ADDITIONAL INFORMATION}

Supplementary information is available for this paper at https://doi.org/10.1038/ s41523-019-0144-4.
Correspondence and requests for materials should be addressed to I.S. or F.J.E.

Reprints and permission information is available at http://www.nature.com/ reprints

Publisher's note Springer Nature remains neutral with regard to jurisdictional claims in published maps and institutional affiliations.

\section{cc) (i)}

Open Access This article is licensed under a Creative Commons Attribution 4.0 International License, which permits use, sharing, adaptation, distribution and reproduction in any medium or format, as long as you give appropriate credit to the original author(s) and the source, provide a link to the Creative Commons license, and indicate if changes were made. The images or other third party material in this article are included in the article's Creative Commons license, unless indicated otherwise in a credit line to the material. If material is not included in the article's Creative Commons license and your intended use is not permitted by statutory regulation or exceeds the permitted use, you will need to obtain permission directly from the copyright holder. To view a copy of this license, visit http://creativecommons. org/licenses/by/4.0/.

(c) The Author(s) 2020 